\title{
ZU EINIGEN INSCHRIFTEN DER DRITTEN ZWISCHENZEIT ${ }^{1}$
}

\author{
[TAFEL X-XVI]
}

VON

\author{
Karl JANSEN-WINKELN \\ Ägyptologisches Seminar der FU \\ Altensteinstr. 33 \\ D-14195 Berlin \\ Allemagne
}

Im folgenden sollen die Inschriften von vier Statuen der 22./23. Dynastie behandelt werden, die bisher teils noch gar nicht, teils nur auszugsweise veröffentlicht waren².

\section{Nr.1: Würfelhocker Kairo JE 36926 (Fig. 1; Taf. X)}

Die Statue stammt aus der Cachette von Karnak (Fundnummer K.64). Sie ist aus grauem Granit und $63 \mathrm{~cm}$ hoch; der Sockel ist $41 \mathrm{~cm}$ breit, $50 \mathrm{~cm}$ tief, $15,5 \mathrm{~cm}$ hoch, der Rückenpfeiler $20 \mathrm{~cm}$ breit. Sie wird im Kellermagazin des Museums aufbewahrt.

Die Figur sitzt auf einem Sockel mit einer Stufe im hinteren Bereich, die Füße sind nicht frei, zeichnen sich aber deutlich ab, wie das bei größeren Würfelhockern in dieser Zeit öfter vorkommt ${ }^{3}$. Die linke Hand liegt flach ausgestreckt auf dem Knie, die rechte hält eine Pflanze. Der Kopf der Statue ist samt dem oberen Teil des Rückenpfeilers weggebrochen. Auch der Sockel ist beschädigt, v.a. an den Kanten. Der Rest der Statue ist gut erhalten.

Auf der Vorderseite ist eine Darstellungen mit Beischriften, oben von einer Himmelshieroglyphe, an den Seiten und unten von Linien begrenzt. Rechts steht eine männliche Person, kahlköpfig, in ein langes Gewand und das Pantherfell gekleidet, in der linken Hand ein Räuchergerät haltend und mit der rechten auf einen kleinen Altar libierend. Ihm zugewandt steht eine Person mit Falkenkopf, die mit einem Schurz bekleidet ist und die Doppelfederkrone mit Sonnenscheibe und Uräus trägt, in der linken Hand ein langes Szepter vor sich haltend, in der herabhängenden rechten ein $n h$-Zeichen.

\footnotetext{
1 Abkürzungen nach Lexikon der Ägyptologie, Bd. 7, 1992, XIII-XXXVIII; außerdem: Äg. Biogr. = K. Jansen-Winkeln, Ägyptische Biographien der 22. und 23. Dynastie (ÄUAT 8), 1985; Spätmittelägyptische Grammatik = K. JansenWinkeln, Spätmittelägyptische Grammatik der Texte der 3. Zwischenzeit (ÄUAT 34), 1996.

${ }^{2}$ Für die Genehmigung zur Publikation und die Wiedergabe der Fotos danke ich für die Nr.1-3 dem ehemaligen Generaldirektor des ägyptischen Museums Kairo, Prof. Dr. M. Saleh, für Nr. 4 seinem Nachfolger Dr. M.A.H Shimy sehr herzlich. Alle Fotos sind vom Museum Kairo aufgenommen worden.

3 Vgl. z.B. Kairo CG 559 oder CG 42189, s. L. Borchardt, Statuen und Statuetten von Königen und Privatleuten (CG), Bd. 2, Berlin 1925, Bl. 94; G. Legrain, Statues et statuettes de rois et de particuliers (CG), Bd. 2, Kairo 1909, pl. LI.
} 
Ansonsten ist die Statue weder dekoriert noch beschriftet, selbst der Rückenpfeiler ist unbeschrieben.

Besitzer:

enh.f-n-Hnzw

Vater:

$N s-p 3-h r-n-t 3-h \underline{h} t$

Großvater:

Hr-3hbjt hm-ntr $n$ Jmn-R ${ }^{e}$ njswt ntrw

$m r$ pr-ḥd $n$ pr-Jmn

hm-ntr jmj 3bd.f n pr-Jmn hr z3 3-nw

ḥm-ntr jmj 3bd.f n pr-Jmn ḥr z3 3-nw

ḥm-ntr jmj 3bd.f n pr-Jmn hrr z3 3-nw

Diese Namen und Titel sind in der Dritten Zwischenzeit sehr geläufig, aber die drei Personen lassen sich keiner der thebanischen Familien dieser Zeit sicher zuordnen. Nach Stil, Dekoration und Personennamen dürfte die Statue in die frühere 22. Dynastie gehören. Sie ist unpubliziert und wird m.W. nur einmal in der Literatur erwähnt ${ }^{4}$.

Beischrift zu Götterfigur:

Ein Königsopfer des Month-Re, des Herrn von Theben, des großen(?)(1) Gottes, des Herrn des Himmels, des Oberhaupts der Beiden Länder.

Beischrift zu Opferndem:

Der Prophet des Amonrasonther, der Vorsteher des Schatzhauses des Hauses des Amun, der Prophet im Monatsdienst des Hauses des Amun in der 3. Phyle 'nh.f-n-Hnzw, gerechtfertigt, der Sohn des Propheten im Monatsdienst des Hauses des Amun in der 3. Phyle Ns-

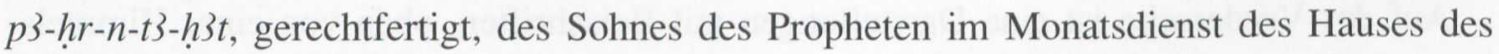
Amun in der 3. Phyle $\mathrm{Hr}$-3hbjt, gerechtfertigt.

\section{ANMERKUNGEN}

1) Das Zeichen hat eine merkwürdige Form, fast wie ein umgekehrtes 8 .

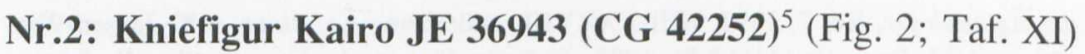

Die Statue stammt aus der Cachette von Karnak (Fundnummer K.286). Sie ist aus Kalzit und 52,5 cm hoch; der Sockel ist $32,4 \mathrm{~cm}$ tief, $22 \mathrm{~cm}$ breit und $6,2 \mathrm{~cm}$ hoch.

${ }^{4} \mathrm{PM} \mathrm{II}^{2}$, p. 150 .

${ }^{5}$ Ursprünglich zur Publikation im Catalogue général durch Ch. Kuentz vorgesehen. 


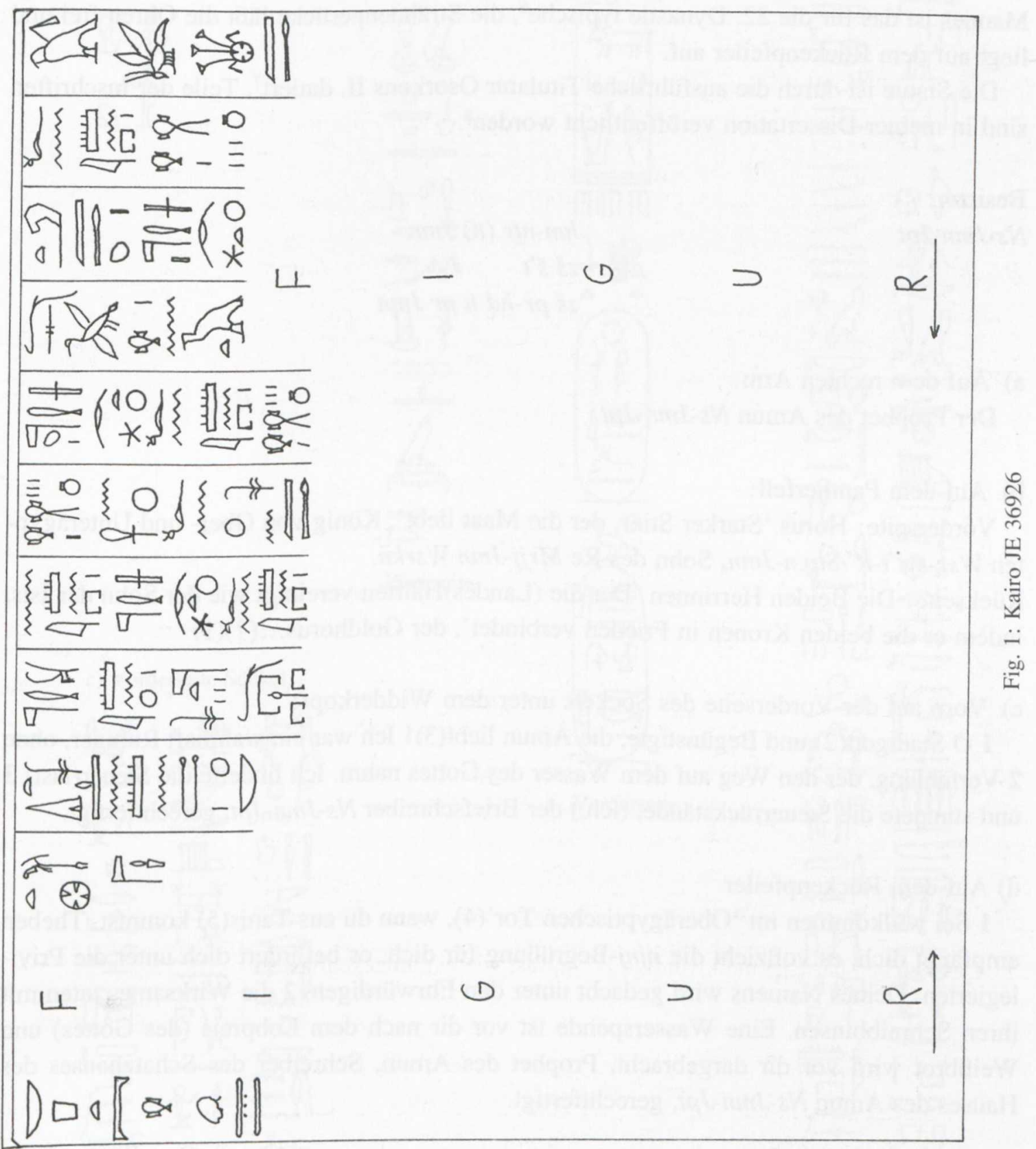


Eine männliche Figur, die über einem bauschigen und faltenreichen Gewand mit abstehenden Ärmeln das Pantherfell trägt, kniet auf einem Sockel und hält in den flach ausgestreckten Händen eine Art Schrein vor sich, auf dem ein Widderkopf ruht. Das Gesicht des Mannes ist das für die 22. Dynastie typische ${ }^{6}$, die Strähnenperücke läßt die Ohren frei und liegt auf dem Rückenpfeiler auf.

Die Statue ist durch die ausführliche Titulatur Osorkons II. datiert ${ }^{7}$. Teile der Inschriften sind in meiner Dissertation veröffentlicht worden ${ }^{8}$.

Besitzer:

Ns-Jmn-Jpt

$$
\begin{aligned}
& \text { hm-ntr (n) Jmn } \\
& z \check{s} \breve{s} t \\
& z \check{s} \text { pr-hִd n pr Jmn }
\end{aligned}
$$

a) Auf dem rechten Arm:

Der Prophet des Amun Ns-Jmn-Jpt.

b) Auf dem Pantherfell:

Vorderseite: Horus 'Starker Stier, der die Maat liebt', König von Ober- und Unterägypten Wsr-m3't-R' Stp.n-Jmn, Sohn des Re Mrjj-Jmn Wsrkn.

Rückseite: Die Beiden Herrinnen 'Der die (Landes)Hälften vereinigt wie der Sohn der Isis, indem er die beiden Kronen in Frieden verbindet', der Goldhorus...(?)(1)

c) Vorn auf der Vorderseite des Sockels unter dem Widderkopf:

1 O Stadtgott(2) und Begünstigte, die Amun liebt(3)! Ich war ein wahrhaft Ruhiger, ohne 2 Verfehlung, der den Weg auf dem Wasser des Gottes nahm. Ich linderte die Steuer(last) 3 und stundete die Steuerrückstände, (ich,) der Briefschreiber Ns-Jmn-Jpt, gerechtfertigt.

d) Auf dem Rückenpfeiler

1 Sei willkommen im 'Oberägyptischen Tor'(4), wenn du aus Tanis(5) kommst. Theben empfängt dich, es vollzieht die njnj-Begrüßung für dich, es befördert dich unter die Privilegierten. Deines Namens wird gedacht unter den Ehrwürdigen, 2 die Wirksames taten mit ihren Schreibbinsen. Eine Wasserspende ist vor dir nach dem Lobpreis (des Gottes) und Weißbrot wird vor dir dargebracht, Prophet des Amun, Schreiber des Schatzhauses des Hauses des Amun Ns-Jmn-Jpt, gerechtfertigt.

${ }^{6}$ S. K. Bosse, Die menschliche Figur ( $\ddot{A} F$ 1), 1936, p. 26.

7 Vgl. dazu auch H. Jacquet-Gordon, JEA 46 (1960), p. 14, no.1,II.

8 Äg. Biogr., p. 272-3; zur bisherigen Literatur s. dort, p. 272. Dazu kommt noch M. Weber, Beiträge zur Kenntnis des Schrift- und Buchwesens der alten Ägypter, Diss. Köln 1967 (1969), p. 46. 
a) Auf dem rechten Arm

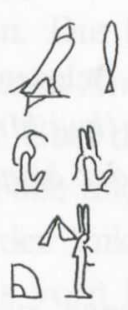

c) Vorderseite Sockel

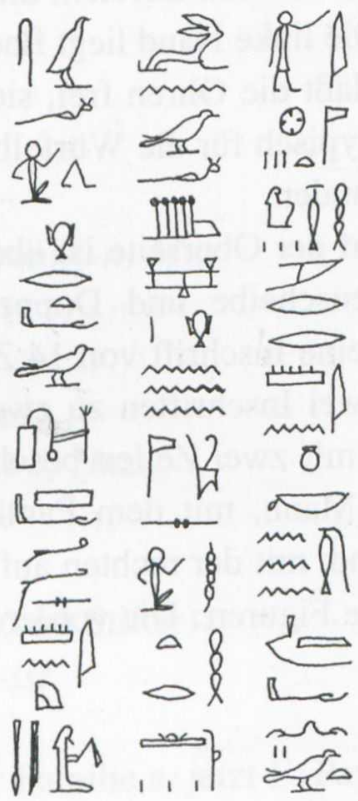

b) Auf dem rechten Arm

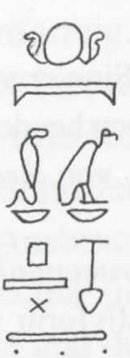

$\frac{1}{a} \prod_{i=0}^{0}$

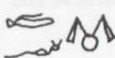

边

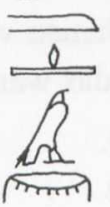

$\rightleftarrows$ sic

Rückseite

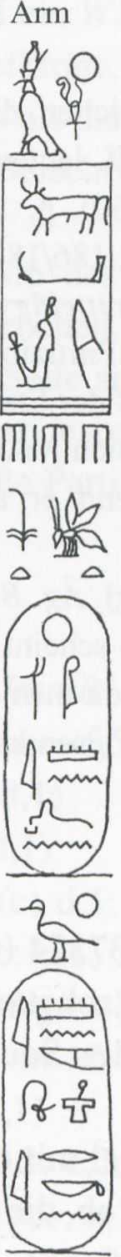

Vorderseite d) Rückenpfeiler $\infty \Lambda$ tरo 119

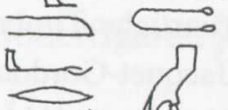
III y

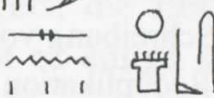
?क्ष तथ $\theta$ $\min \Omega \Lambda$ $\min \pi$ 1 तर $\infty$ odn है का एक ๑) monl IIItis

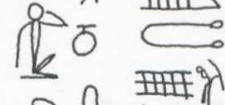
बी 3 ?<smiles>COc1cccc(C(C)(C)C)c1</smiles>

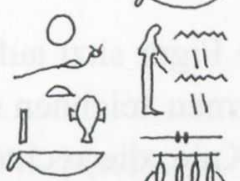
RoP MIN mर

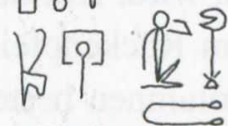
im 14 要, 药 
ANMERKUNGEN

1) Unter $H r n b w$ zwei unförmige Zeichen, die keinen Sinn ergeben. In den anderen Belegen für die ausführliche Titulatur Osorkons II. lautet der entsprechende Titel meist wr phtj (hwj Mntjw), s. H. Jacquet-Gordon, JEA 46 (1960), p. 14, n.1; J. von Beckerath, Handbuch der ägyptischen Königsnamen (MÄS 49), 1999, p. 186/187.

2) Zur Schreibung von nwtj s. Spätmittelägyptische Grammatik, §29; 175.

3) Zur Reduplikation bei passivem Partizip und Relativform von $m r j$ vgl. ibid., §200. Eine ganz ähnliche Anrufung findet sich auf der Statue gleichen Typs Kairo CG 42214, s. Äg. Biogr., 109; 110 (3); 488. Man beachte, daß sich Ns-Jmn-Jpt auf der Vorderseite dem Gott und den anderen Statuenbesitzern vorstellt, während er auf der Rückseite selbst angeredet und willkommen geheißen wird.

4) Eine Bezeichnung für Theben, vgl. Äg. Biogr., 288-9 (3).

5) Eine solche Schreibung $\left(\underline{D}-{ }^{e} n(\underline{d})\right)$ scheint für Tanis sonst nicht belegt zu sein. Vielleicht handelt es sich um eine Schriftspielerei, da man hier den Namen von Tanis auch als W3djt ${ }^{\mathrm{C}} \underline{d}(t)$,,heile Uto“ bzw. „Uto ist heil“ ( $\left.{ }^{(} d(. t j)\right)$ lesen könnte, und das wäre für eine unterägyptische Residenzstadt ja recht passend.

\section{Nr.3: Würfelhocker Kairo JE 37374 (CG 42254) ${ }^{9}$ (Taf. XII-XV)}

Die Statue stammt aus der Cachette von Karnak (Fundnummer K.389). Sie ist aus Kalkstein und 49,5 cm hoch; der Sockel ist $35 \mathrm{~cm}$ tief, 24,2 cm breit und 4-4,5 cm hoch.

Die Figur sitzt auf einem Sockel mit einem ovalen Kissen im hinteren Bereich, die Körperformen zeichnen sich deutlich ab, die Füße sind nicht frei. Die linke Hand liegt flach auf dem Knie, die rechte hält eine Pflanze. Die Strähnenperücke läßt die Ohren frei, sie liegt auf dem (ziemlich breiten) Rückenpfeiler auf. Das Gesicht ist typisch für die Würfelhocker der 22./23. Dynastie ${ }^{10}$, der Bart ist mit dem Körperblock verbunden.

Die Statue ist folgendermaßen beschriftet und dekoriert: Auf der Oberseite ist über den Händen jeweils eine Kartusche eingraviert, die von Sonnenscheibe und Doppelfeder gekrönt wird. Auf der rechten und der linken Seite ist jeweils eine Inschrift von 14 Zeilen, auf dem Rückenpfeiler finden sich fünf Kolumnen, die aus zwei Inschriften zu zwei und drei Kolumnen bestehen. Auf der Vorderseite sind die Arme mit zwei Zeilen beschriftet, darunter ist eine Darstellung: Rechts steht ein kahlköpfiger Mann, mit dem Pantherfell bekleidet, in der linken Hand einen Räucherarm vor sich haltend, mit der rechten auf einen Altar vor sich libierend. Ihm zugewandt stehen zwei männliche Figuren: Die vordere trägt

\footnotetext{
9 S.o., Fußnote 5.
}

${ }^{10}$ S.o., Fußnote 6. 
die Doppelfederkrone und hält in der linken Hand ein W3s-Szepter, in der rechten ein ' $n h$ Zeichen. Die hintere ist mumienförmig, mit Atefkrone, und hält in den über der Brust gekreuzten Armen Wedel und $h q$ 3-Szepter. Anders als die vordere Figur steht sie auf einem Sockel. Über dem Opferer stehen fünf Kolumnen Beischrift, über den Götterfiguren jeweils zwei. Füße und Sockel sind unbeschriftet.

An der linken Hand ist ein Ausbruch unterhalb des kleinen Fingers, die Ecken des Sockels vorn links und hinten rechts sind abgebrochen, daneben gibt es einige kleinere Bestoßungen. Auf der Vorderseite und der linken Seite sind die Inschriften teilweise abgeschliffen und dadurch an manchen Stellen schwer lesbar, die rechte Seite der Figur ist durch Wasser und Salz schwer beeinträchtigt, große Partien der Inschrift sind zerstört oder unlesbar.

Besitzer:

$J w \cdot f-3$

hm-ntr n Jmn-R ${ }^{\mathrm{e}}$ njswt-ntrw (b,1-2; c; e, 1; f,1) / hm-ntr n Jmn (f,5) / hmm-ntr n Jmn m Jpt-swt (d,1) $j r j-p^{t} t(\mathrm{c} ; \mathrm{d}, 1)$

$h 3 t j-{ }^{\circ}(\mathrm{c} ; \mathrm{d}, 1)$

htmtj-bjtj (c; d,1; f,1)

$s m r w^{i} t j(\mathrm{c} ; \mathrm{d}, 1 ; \mathrm{f}, 1)$

hm-ntr $n$ Mntw nb Jwnw-šm $w$ (b,2; d,1)

mr k3t n mnw nb n nbw n pr-Jmn (b,2; d,1)

žs njswt (f,1)

$r w d w$ ' 3 (d,2; e,2; f,5)

hsf $n n w t(\mathrm{~d}, 2 ; \mathrm{e}, 2 ; \mathrm{f}, 5)$

hmm-ntr 3-nw n Mwt nbt Jšrw [ ... ] $(\mathrm{e}, 1)$

jdnw $p r-h \underline{d} \underline{n} n n b t 3 w j(\mathrm{e}, 2)$

Vater:

$P 3(-n)-m w(-n)-M w t$

$m j-n n(\mathrm{~d}, 2)$

$j t j-n \underline{t} r \mathrm{mrjj}(-n \underline{t} r)(\mathrm{c})$

[ ] $(\mathrm{e}, 2)$

Mutter:

T3(-nt)-dj-Hnzw

$n b t-p r(\mathrm{e}, 2)$

$j[$ hyt $n] J m n-R^{e}(\mathrm{e}, 2)$

Sohn (und Stifter):

$H r-z 3-3 s t$

hmm-ntr n Jmn m Jpt-swt (b,1)

Zur Familie s. jetzt F. Payraudeau, RdE 54 (2003), 134-5 (3). 
Die Statue ist durch die Kartuschen des (oberägyptischen) Königs Harsiese datiert ${ }^{11}$. Sie ist unpubliziert, ihre Texte waren aber schon teilweise bekannt. Große Teile davon (b, d und f) hatte schon K. Sethe für das Wörterbuch aufgenommen ${ }^{12}$. Nach dieser Abschrift sind sie einigemal zitiert worden ${ }^{13}$, und in meiner Dissertation habe ich größere Teile der Inschriften d (linke Seite) und f (Rückseite) behandelt ${ }^{14}$. Auch in meiner Sammlung von "Sentenzen“ habe ich die Inschrift mehrfach herangezogen ${ }^{15}$.

a) Auf der Oberseite (Fig.6)

rechts: $H \underline{d}-h p r-R^{e} S t p \cdot n-R^{e}$

links: Mrjj-Jmn $\mathrm{Hr}$-z3-3st

b) Auf den Knien (Fig.3)

1 Gemacht von seinem Sohn, um seinen Namen am Leben zu erhalten, dem Propheten des Amun in Karnak $H r-z 3-3 s t$, dem Sohn des Propheten des 2 Amonrasonther, des Propheten des Month, des Herrn des oberägyptischen Heliopolis, des Baumeisters aller Denkmäler von Gold des Hauses des Amun(1) Jw.f-'3.

c) Vorderseite, Beischriften (Fig.3)

$\mathrm{Zu}$ Amun: Sprechen von Worten durch Amun-Re, den Herrn der Throne der Beiden Länder, den Ersten von Karnak.

Zu Osiris: Sprechen von Worten durch Osiris, den Ersten des Westens, den Herrn des Himmels.

Zu Opferer: Der Osiris, der Prophet des Amonrasonther, der Erbfürst und Graf(2), der königliche Siegler, der einzigartige Freund $J w . f_{-}{ }^{-} 3$, der Sohn des Gottesvaters und Mrjj($n t r)$ P3-mw-mwt(3), gerechtfertigt.

d) Linke Seite (Fig.4)

1 Der Prophet des Amun in Karnak, der Prophet des Month, des Herrn des oberägyptischen Heliopolis, der Erbfürst und Graf, der königliche Siegler, einzigartige Freund, der

${ }^{11}$ Vgl. auch JEA 81 (1995), p. 134

${ }^{12}$ Kairo Wb Nr.49, Zettel $<51>-<60>$.

${ }^{13}$ Z.B. von Erman, $N G$, $\S 83$ (zu d,10); $\$ 113$ (zu d,7); F. Hintze, Untersuchungen zu Stil und Sprache neuägyptischer Erzählungen, 1950, p. 136, n.1 (zu d,10-11); G. Griffiths, Harvard Theological Review 53 (1960), p. 220 (zu d,8); P. Vernus, in: Cahiers de Karnak VI (1973-77), 1980, p. 224 (zum Titel $r w d w$ ' 3 hsf $n$ nwt).

${ }_{14} \ddot{A g}$. Biogr., 263-6 (B 10), leider für Text d (linke Seite) mit falscher Zeilenzählung: die in Äg. Biogr. auf p. 264 zitierten Z.6-13 sind in Wirklichkeit d,7-14! Meine Zitate dieser Inschrift in K. Jansen-Winkeln, Text und Sprache in der 3. Zwischenzeit (ÄUAT 26), 1994, (Sigel B/3.3.34, vgl. p. 360) und Spätmittelägyptische Grammatik (vgl. p. 550) sind entsprechend zu korrigieren.

${ }^{15}$ K. Jansen-Winkeln, Sentenzen und Maximen, 1999 (auch hier mit falscher Zeilenzählung): 50 (A.1.c.1: d,6); 51 (A.1.c.7: d,6); 53 (A.1.c.26: d,8-9; A.1.c.27: d,9); 70 (A.2.h.1: d,7-8); 88 (A.4.b.22: d,6-7); 90 (A.4.d.3: d,7). 


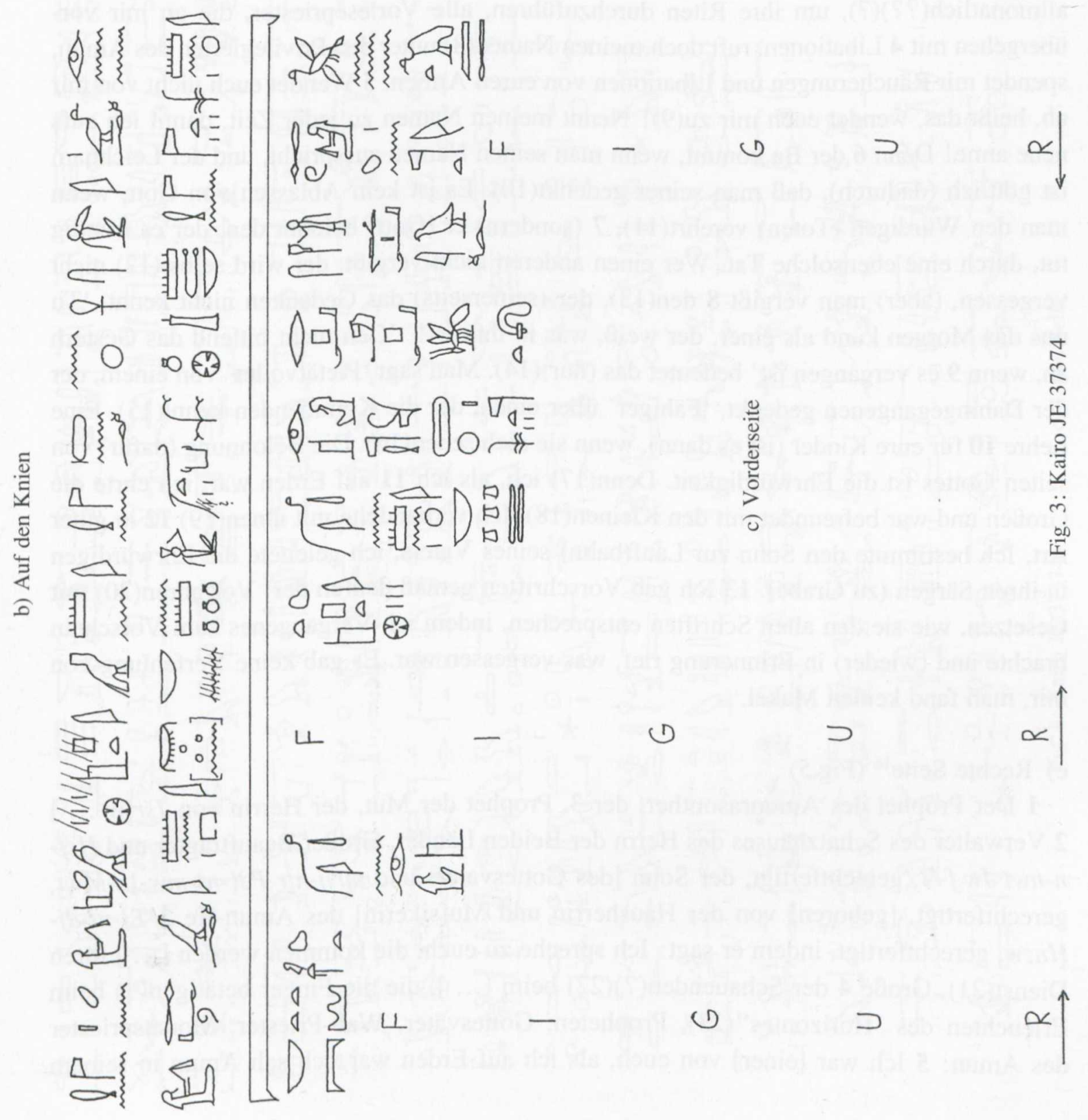


Baumeister aller Denkmäler(4) von Gold des Hauses des Amun, 2 der Große Beauftragte und $H s f-n-n w t(5) J w . f-{ }^{e}$, gerechtfertigt, der Sohn des gleichrangigen P3-n-mw-n-Mwt, gerechtfertigt, indem er sagt: O Propheten und Gottesväter, die in Karnak sind, Wab-Priester, 3 Monatsdiener(?)(6), die beim Gott eintreten, die hinausgehen und zurückkommen allmonatlich(??)(7), um ihre Riten durchzuführen, alle Vorlesepriester, die an mir vorübergehen mit 4 Libationen, ruft doch meinen Namen(8) unter den Privilegierten des Amun, spendet mir Räucherungen und Libationen von euren Armen. 5 Wendet euch nicht von mir $\mathrm{ab}$, heißt das, wendet euch mir zu(9)! Nennt meinen Namen zu jeder Zeit, damit ich aufs neue atme! Denn 6 der Ba kommt, wenn man seinen Namen ausspricht, und der Leichnam ist göttlich (dadurch), daß man seiner gedenkt(10). Es ist kein Ablassen von Gott, wenn man den Würdigen (Toten) verehrt(11), 7 (sondern) er [Gott] belohnt den, der es freudig tut, durch eine ebensolche Tat. Wer einen anderen nicht vergißt, der wird selbst(12) nicht vergessen, (aber) man vergißt 8 den(13), der (seinerseits) das Gedenken nicht kennt. 'Tu uns das Morgen kund als einer, der weiß, was in ihm ist!' 'Geh nicht bittend das Gestern an, wenn 9 es vergangen ist' bedeutet das (nur)(14). Man sagt 'Pietätvoller' von einem, der der Dahingegangenen gedenkt, 'Fähiger' über einen, der die Kommenden kennt(15). Eine Lehre 10 für eure Kinder (ist es dann), wenn sie dich sehen(16). Die Belohnung (dafür) von seiten Gottes ist die Ehrwürdigkeit. Denn(17) ich, als ich 11 auf Erden war, ich ehrte die Großen und war befreundet mit den Kleinen(18). Ich verhandelte mit ihnen(19) 12 in guter Art. Ich bestimmte den Sohn zur Lauf(bahn) seines Vaters, ich geleitete die Ehrwürdigen in ihren Särgen (zu Grabe). 13 Ich gab Vorschriften gemäß den(en der) Vorfahren(20) mit Gesetzen, wie sie den alten Schriften entsprechen, indem ich Vergangenes zum Vorschein brachte und (wieder) in Erinnerung rief, was vergessen war. Es gab keine Verfehlung von mir, man fand keinen Makel.

e) Rechte Seite ${ }^{16}$ (Fig.5)

1 Der Prophet des Amonrasonther, der 3. Prophet der Mut, der Herrin von J̌̌r [w ... ] 2 Verwalter des Schatzhauses des Herrn der Beiden Länder, Großer Beauftragter und Hsf$n$-nwt Jw.f- ${ }^{3}$, gerechtfertigt, der Sohn [des Gottesvaters und mrjj-ntr P3(-n)-mw-]n-Mwt, gerechtfertigt, [geboren] von der Hausherrin und Mu[sikerin] des Amun-Re 3 T3-nt-dj$H n z w$, gerechtfertigt, indem er sagt: Ich spreche zu euch, die kommen werden [... ] ihren Dienst(21), Große 4 der Schauenden(?)(22) beim [... ], die die Finger betätigen(?) beim Erleuchten des 'Horizontes“(23), Propheten, Gottesväter, Wab-Priester, Monatspriester des Amun: 5 Ich war [einer] von euch, als ich auf Erden war, ich sah Amun in seinem

\footnotetext{
${ }^{16}$ Diese Inschrift ist in großen Teilen so schwer beschädigt, daß eine zusammenhängende Übersetzung nur für wenige Passagen möglich ist. An einigen Stellen scheint noch etwas mehr zu erkennen zu sein, aber es ist für eine halbwegs sichere Wiedergabe zu undeutlich. Vielleicht wäre eine weitere Vervollständigung möglich, wenn man die Inschrift längere Zeit unter günstigen Lichtverhältnissen studieren könnte.
} 


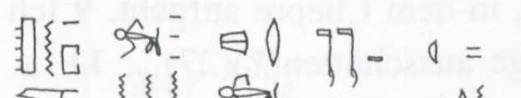

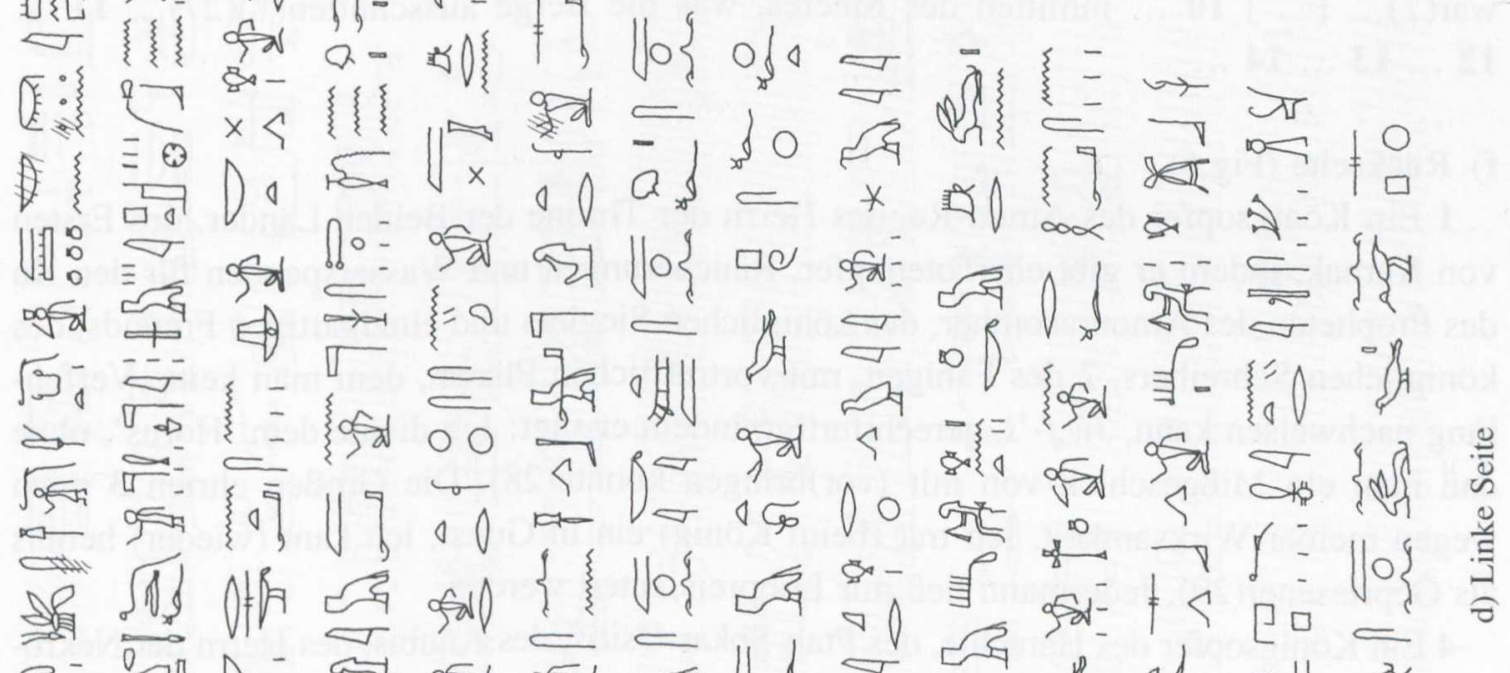

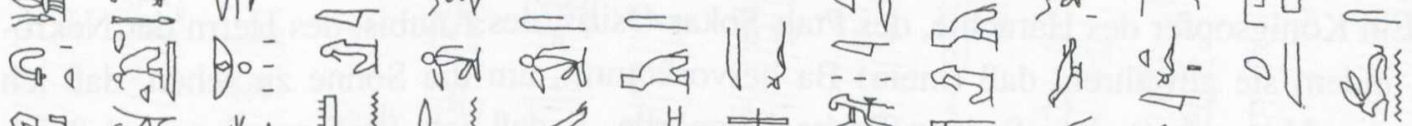

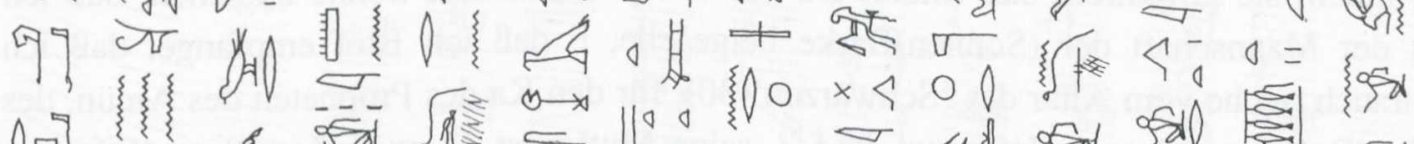

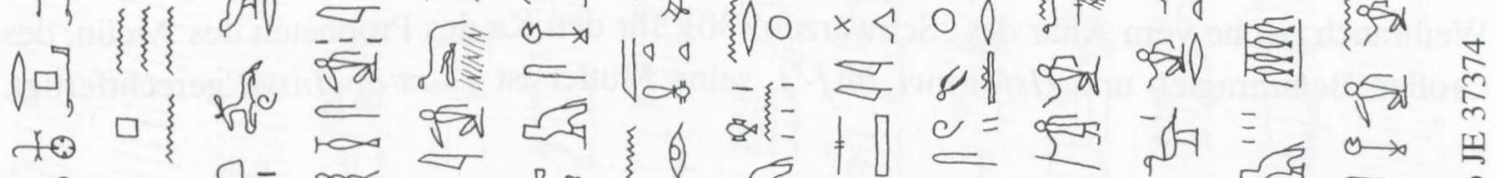

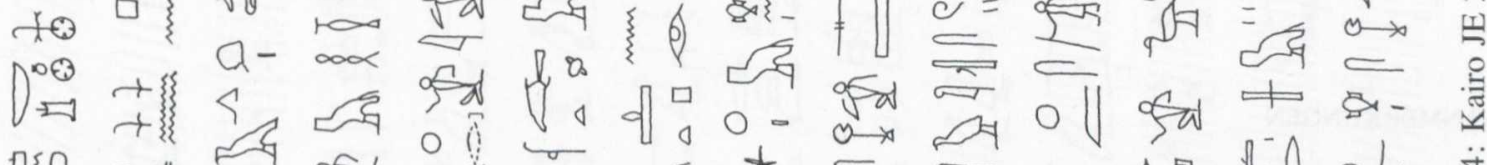

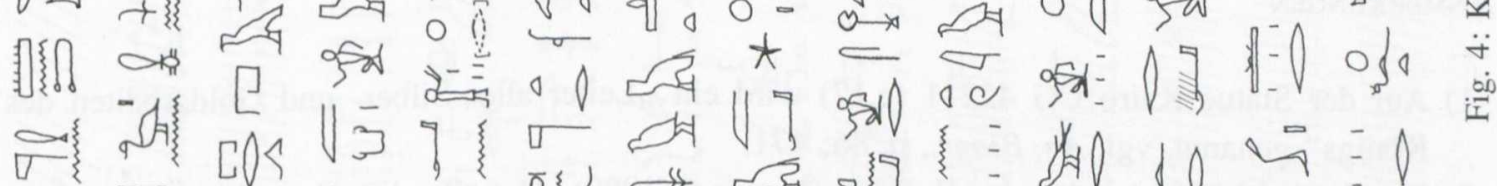

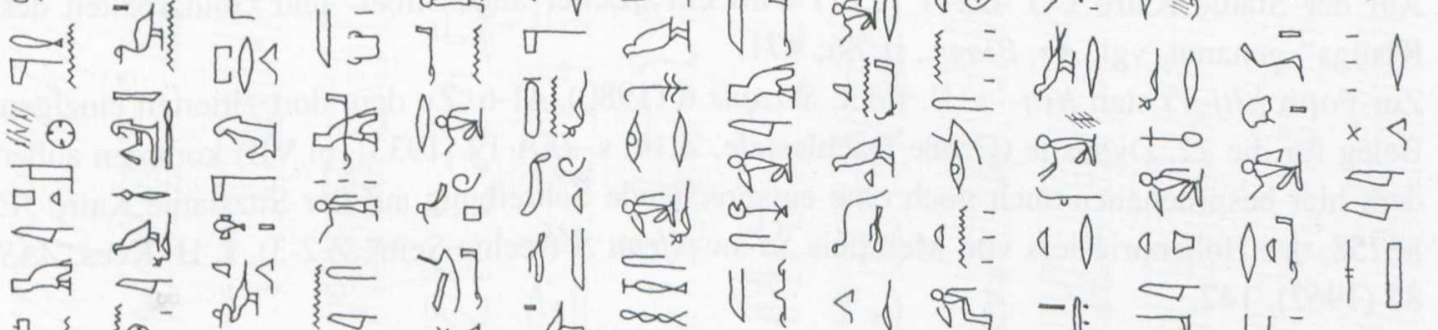

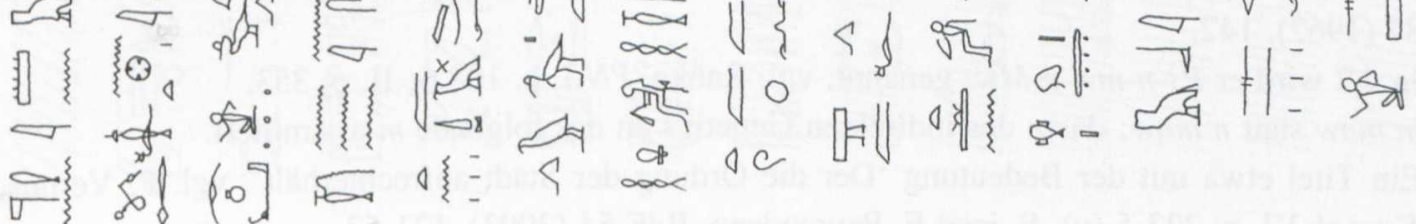


'Horizont'. Mich entsandte $\mathbf{6}$ der Herrscher entsprechend seinem Wunsch in allen bedeutenden Aufträgen, er beförderte mich unter den Höflingen(24). Seine Majestät gab mich 7 [... ] Amonrasonther bezüglich der Arbeit(?) in Gold(25), während er Vorschriften gab wie Ptah(26). 8 [... ]... um zu erscheinen (im) 'Horizont', in dem Chepre aufgeht. 9 Ich $w a r(?) \ldots[\ldots] \mathbf{1 0} \ldots$ inmitten des Meeres, was die Berge ausschütten(?)(27).. $11 \ldots$ $12 \ldots 13 \ldots 14 \ldots$

\section{f) Rückseite (Fig.6)}

1 Ein Königsopfer des Amun-Re, des Herrn der Throne der Beiden Länder, des Ersten von Karnak, indem er gibt ein Totenopfer, Räucherungen und Wasserspenden für den Ka des Propheten des Amonrasonther, des königlichen Sieglers und einzigartigen Freunds, des königlichen Schreibers, 2 des Fähigen, mit vortrefflichen Plänen, dem man keine Verfehlung nachweisen kann, $J w . f-{ }^{-} 3$, gerechtfertigt, indem er sagt: Ich diente dem 'Horus', ohne daß man ein Mißgeschick von mir (vor)bringen konnte(28). Die Großen ehrten 3 mich wegen meiner Wirksamkeit. Ich trat (beim König) ein in Gunst, ich kam (wieder) heraus als Gepriesener(29). Jedermann ließ mir Lobpreis zuteil werden.

4 Ein Königsopfer des Harachte, des Ptah-Sokar-Osiris, des Anubis, des Herrn der Nekropole, indem sie gewähren, daß (mein) Ba hervorkommt, um die Sonne zu sehen, daß ich mich der Mannschaft der (Sonnen)Barke beigeselle, $5 \mathrm{da} \beta$ ich Brot empfange, daß ich Weihrauch rieche vom Altar des 'Schwarzen'(30), für den Ka des Propheten des Amun, des Großen Beauftragten und $H s f-n-n w t J w . f^{-}$'3, seine Mutter ist $T 3-n t-d j-H n z w$, gerechtfertigt.

\section{ANMERKUNGEN}

1) Auf der Statue Kairo CG $42211(\mathrm{e}, 17)$ wird ein „Leiter aller Silber- und Goldarbeiten des Königs" genannt, vgl. Äg. Biogr., p. 86; 471.

2) Zur Form $h 3 t j-p^{\prime} t$ statt $h 3 t j-{ }^{\circ}$ s. E. Edel, Serapis $6(1980), 41-6$. Zu dem dort zitierten einzigen Beleg für die 22. Dynastie (Große Dachlestele, Z.16, s. JEA 19 [1933], pl.VII) kommen außer dem hier besprochenen auch noch eine entsprechende Schreibung auf der Sitzstatue Kairo JE 86758, des Hohenpriesters von Memphis $\breve{S} d$-sw-Nfrtm A (rechte Seite, Z.2-3), s. H. Kees, Z̈̈S 87 (1962), 142.

3) In d,2 wird er $P 3-n-m w-n-M w t$ genannt, vgl. Ranke, $P N$ I, p. 108,6; II, p. 353.

4) $m m n w$ statt $n m n w$; das $n$ des indirekten Genetivs an das folgende $m$ assimiliert.

5) Ein Titel etwa mit der Bedeutung 'Der die Ordung der Stadt aufrechterhält', vgl. P. Vernus, Karnak VI, p. 223-5 (y). S. jetzt F. Payraudeau, RdE 54 (2003), 131-53.

6) Vgl. zu diesem Titel $\ddot{A} g$. Biogr., p. 187-8 (4) und CdE 67 (1992), p. 247.

7) Sehr fraglich; das Wörterbuch (I, 56, 12) versteht das Hapax legomenon in Theben“, aber ein solches Fest („Bohnenfest“??) scheint nicht bekannt zu sein. An entspre- 


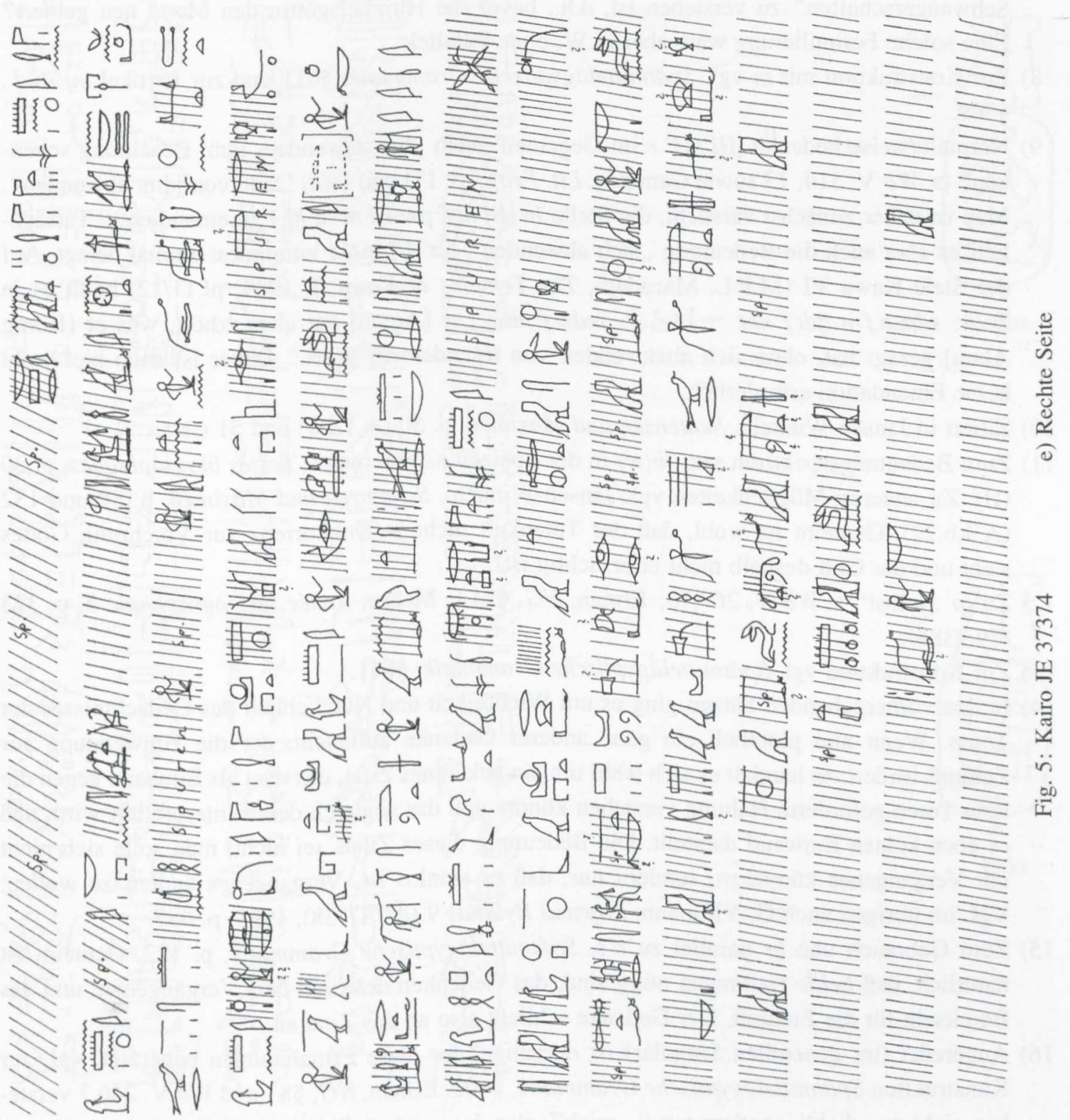


chenden Stellen in den Anrufen an die Lebenden heißt es sonst z.B. (prj h3j) r-tp $3 b d$,am Beginn des Monats“, vgl. K. Jansen-Winkeln, Biographische und religiöse Inschriften der Spätzeit (ÄUAT 45), 2001, 369 (15,d,3); 410 (30, a,3) oder (prj h3j) m nmtt j’̣ ,,gemäß dem Gang des Mondes“, s. ibid., 408 (29, a,5) u.ö. Ob hier vielleicht wörtlich ,,jedesmal bei den Schwangerschaften“ zu verstehen ist, d.h., bevor die Himmelsgöttin den Mond neu gebiert? Eine solche Formulierung wäre aber m.W. ohne Parallele.

8) Zur Konstruktion mit $m$ vgl. Spätmittelägyptische Grammatik, §611 und zur Partikel mj ibid., $\$ 369$.

9) Normalerweise bedeutet $d 3 j \mathrm{j} 3^{\mathrm{C}} r$ im Gegenteil „sich jdm. zuwenden, jdm. Beachtung schenken“ (s. Wb V, 514, 13 sowie Caminos, Lit. Frag., p. 17) und ' $n(n)$,,sich von jdm. abwenden“. Man ist daher zunächst versucht, die Stelle in $\underline{d} 3 j m 3^{c} p w r . j m$ $n h r . j$ zu emendieren. Tatsächlich ist aber auch die Bedeutung ,sich abwenden“ für $d 3 j m 3^{\circ} r$ zumindest einmal belegt. Auf der Stele Kawa VI (M.F.L. Macadam, The Temples of Kawa, I, 1949, pl.11/12) heißt es in

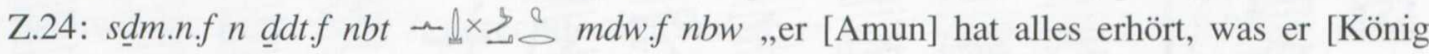
Alara] gesagt hat, ohne sich abzuwenden von irgendeinem Wort." Daher ist auch hier wohl keine Emendation erforderlich.

10) Zitiert in Jansen-Winkeln, Sentenzen und Maximen, p. 50 (A.1.c.1) und 51 (A.1.c.7).

11) Zum Bedeutungsspektrum von $3 b(w)$ in der Spätzeit s. Vercoutter, Textes biographiques, p. 30 (H). Zu anderen Möglichkeiten vgl. Jansen-Winkeln, Sentenzen und Maximen, p. 88 und 132 (A.4.b.22). Gemeint ist wohl, daß der Totenkult nicht in Konkurrenz zur Verehrung Gottes steht und der Gott deshalb nicht eifersüchtig ist.

12) $\mathrm{Zu} t p$ „selbst“ s. $W b \mathrm{~V}, 267,16$; Erman, $N G, \S 113$; Meeks, Année lexicographique 3, p. 323 (79.3384).

13) Zur Konstruktion vgl. Spätmittelägyptische Grammatik, §611.

14) In den vorhergehenden Sätzen ging es um Wichtigkeit und Nützlichkeit des Gedächtnisses der Toten. Wenn nun plötzlich ein ganz anderer Gedanke auftaucht, der die Hinwendung zur Zukunft fordert, so handelt es sich wohl um ein bekanntes Zitat, das man als Einwand gegen die vom Toten geforderte Haltung verstehen könnte und das sogleich derart interpretiert wird, daß es eben keinen Einwand darstellt. Die Bedeutung dieses Zitats sei nicht, man solle sich nicht um Vergangenes kümmern, sondern nur, daß es sinnlos ist, Vergangenes ändern zu wollen. Vgl. im übrigen auch G. Vittmann, Papyrus Rylands 9 (ÄUAT 38), 1998, p. 487.

15) Zum Gebrauch von $h r$ parallel zu $r$ s. Spätmittelägyptische Grammatik, p. 182. Gemeint ist natürlich, daß beide Haltungen nötig sind, das Gedenken des (und der) Vergangenen, und das Bereitsein für die Zukunft. Der Gedanke schließt also an das Zitat an.

16) Angeredet (im generellen Singular) ist derjenige, der diese Ermahnungen beherzigt, vgl. zur Konstruktion Spätmittelägyptische Grammatik, $\S 602$. Erman, $N G, \S 83$ und $W b$ V, 246,7 verstehen nicht $t w$, dich“, sondern $t w(. j)$,,mich“, also das „späte“ Objektspronomen (wie im Demotischen). Dieses Pronomen ist allerdings im Spätmittelägyptischen der Dritten Zwischenzeit sonst nirgends bezeugt, und die 2. Person scheint mir auch besser in den belehrenden Kontext zu passen. 


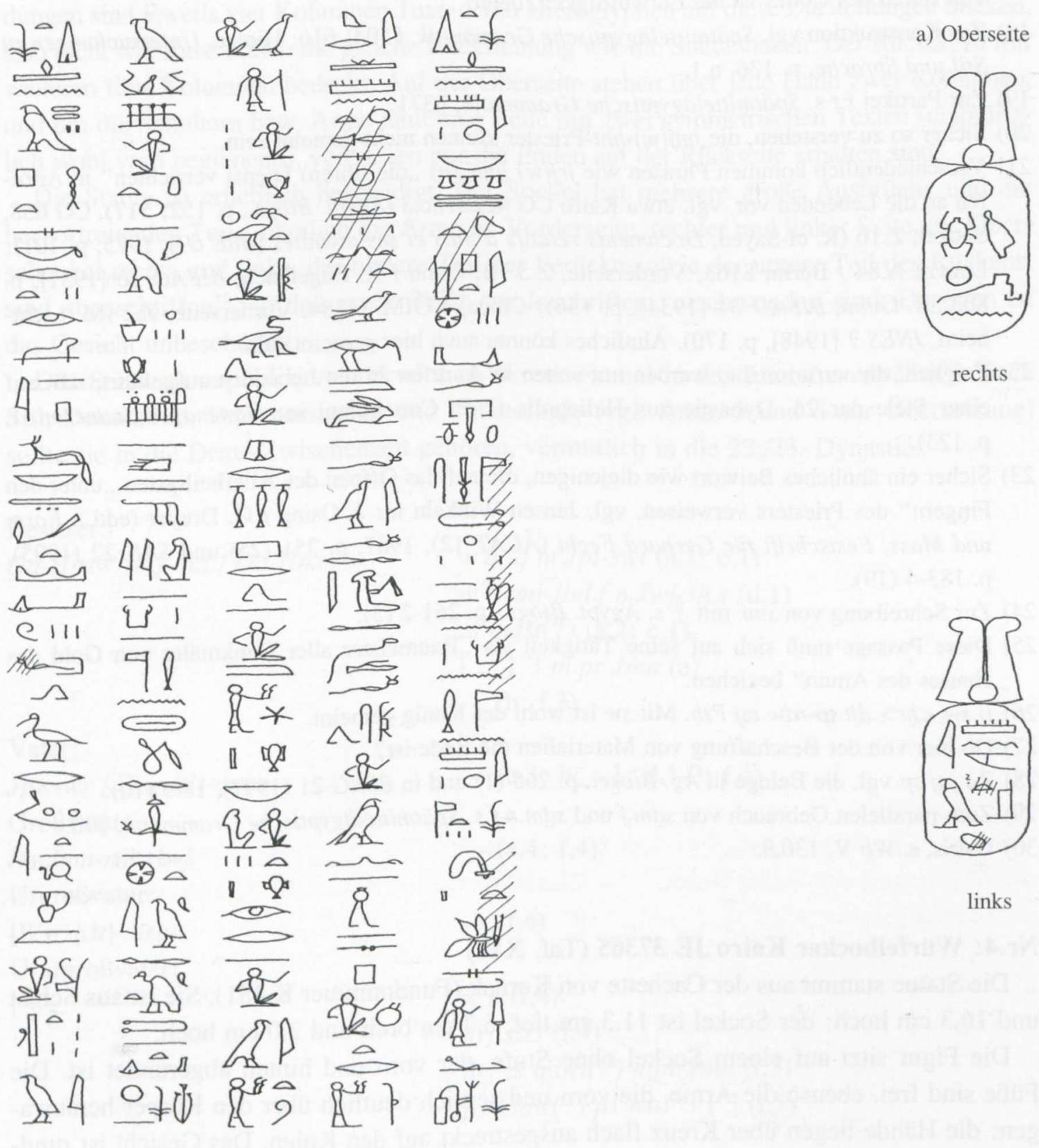

f) Rückseite

Fig. 6: Kairo JE 37374 
17) Entweder liegt hier ein Gedankensprung vor: „Mir ist diese Belohnung zuteil geworden, denn... “, oder der vorhergehende Satz ist in der 1. Person zu verstehen: „(Meine) Belohnung von seiten des Gottes ist die Ehrwürdigkeit, denn..."

18) Zur Konstruktion vgl. Spätmittelägyptische Grammatik, §504; 616; Hintze, Untersuchungen zu Stil und Sprache, p. 136, n.1.

19) Zur Partikel r.f s. Spätmittelägyptische Grammatik, §371.

20) Sicher so zu verstehen, die $j m j(w)$-hnt-Priester können nicht gemeint sein.

21) Verschiedentlich kommen Phrasen wie jrj(w) hnt(.sn) „die (ihren) Dienst verrichten“ in Anrufen an die Lebenden vor, vgl. etwa Kairo CG 42227, c,3 (Ägypt. Biogr., p. 152; 517), CG 658, Sockel, Z.16 (R. el-Sayed, Documents relatifs à Sais et ses divinités [BdE 69], 1975, p. 101); Louvre A.84 / Berlin 8163, Vorderseite, Z.5 (B. Gunn / R. Engelbach, BIFAO 30 [1931], p. 803; B. Gunn, BIFAO 34 [1934], p. 136); Chicago OIM 14284, Vorderseite, Z.2 (M. Lichtheim, JNES 7 [1948], p. 170). Ähnliches könnte auch hier gemeint sein.

22) Fraglich, die $w r(w)-m 3(w)$ werden nur selten in Anrufen an die Lebenden aufgeführt, z.B. auf einer Stele der 26. Dynastie aus Heliopolis (J.-P. Corteggiani in: Hommages Sauneron, I, p. 123).

23) Sicher ein ähnliches Beiwort wie diejenigen, die auf das Öffnen des Allerheiligsten ,unter den Fingern“ des Priesters verweisen, vgl. Jansen-Winkeln in: J. Osing / G. Dreyer (edd.), Form und Mass, Festschrift für Gerhard Fecht (ÄUAT 12), 1987, p. 251 (25) und SAK 22 (1995), p. 183-4 (19).

24) Zur Schreibung von smr mit $\check{\S}$ s. Ägypt. Biogr., p. 261-2 (3).

25) Diese Passage muß sich auf seine Tätigkeit als „Baumeister aller Denkmäler von Gold des Hauses des Amun" beziehen.

26) $t j s w<h r>d j t$ tp-rdw mj Pth. Mit $s w$ ist wohl der König gemeint.

27) $\mathrm{Ob}$ hier von der Beschaffung von Materialien die Rede ist?

28) Zu jnj zp vgl. die Belege in $\ddot{A} g$. Biogr., p. 265 (1) und in BSEG 21 (1997), 18-9 (10).

29) Zum parallelen Gebrauch von $s \underline{d m} . f$ und $s \underline{d m . n . f}$ s. Spätmittelägyptische Grammatik, $\$ 90$.

30) Osiris, s. $W b \mathrm{~V}, 130,8$.

\section{Nr.4: Würfelhocker Kairo JE 37365 (Taf. XVI)}

Die Statue stammt aus der Cachette von Karnak (Fundnummer K.481). Sie ist aus Schist und 16,3 cm hoch; der Sockel ist $11,3 \mathrm{~cm}$ tief, $6,7 \mathrm{~cm}$ breit und 2,0 $\mathrm{cm}$ hoch.

Die Figur sitzt auf einem Sockel ohne Stufe, der vorn und hinten abgerundet ist. Die Füße sind frei, ebenso die Arme, die vorn und seitlich deutlich über den Körper herausragen; die Hände liegen über Kreuz flach ausgestreckt auf den Knien. Das Gesicht ist rundlich, die Strähnenperücke läßt die Ohren frei. Die Statue hat keinen Rückenpfeiler.

Die Vorderseite ist mit einer Abbildung der Sokarbarke dekoriert, die auf einem Tragegestell ruht. Über dem Bug sind zwei Kolumnen Beischrift, über dem Heck eine. Auf der rechten Seite (im hinteren Bereich) steht auf einem Sockel eine mumienförmige Gestalt mit 
oberägyptischer Krone, in beiden Händen Wedel sowie W3s- und hq3-Szepter haltend. Auf der linken Seite befindet sich an entsprechender Stelle der 'Abydosfetisch'17. Vor beiden Abbildungen sind jeweils vier Kolumnen Text, deren Hieroglyphen auf diese Darstellungen blicken, also nicht wie sonst üblich die gleiche Blickrichtung wie die Statue haben. Der Rücken ist mit weiteren fünf Kolumnen bedeckt. Auf der Oberseite stehen über jede Hand zwei Kolumnen, und um die Schultern bzw. Arme läuft eine Zeile mit zwei symmetrischen Texten (ursprünglich wohl vorn beginnend), von denen nur die Enden auf der Rückseite erhalten sind.

Die Statue ist erheblich beschädigt. Der Sockel hat mehrere große Ausbrüche und die hervortretenden Teile, nämlich die Arme an Vorderseite, rechter und linker Seite, die Oberschenkel rechts und links, der hintere Teil der Perücke sowie der untere Teil des Rückens, sind abgeschliffen ${ }^{18}$, so daß große Teile der Inschriften verschwunden sind. Dagegen ist das Gesicht unbeschädigt.

Das Stück ist unpubliziert und wird m.W. nur einmal in der Literatur erwähnt ${ }^{19}$. Nach Stil, Dekoration, Personennamen und Phraeologie (vgl. Anm. 5 und 6 zur Übersetzung) sollte sie in die Dritte Zwischenzeit gehören, vermutlich in die 22./23. Dynastie.

Besitzer:

Dd-Hnzw-jw.f- $n h$ / D d-Hnzw

$$
\begin{aligned}
& \left.w^{\mathrm{c}} b^{\mathrm{e}} q \mathrm{~m} \text { Jpt-swt (a,1; c, } 1\right) \\
& w^{e} b \text { jmj-3bd.f } n \text { Jwj-3h.s (d,1) } \\
& z \check{s} m \underline{d} 3 t(?)(\mathrm{a}, 2 ; \mathrm{c}, 1) \\
& \text { [ ... ] } 3 \text { m pr Jmn (e) } \\
& \text {-.- (b;f,3) }
\end{aligned}
$$

Vater:

Jpw-wr / Wsr-ḩ3t-msjw

--- $(a, 3 ; b ; c, 1 ; d, 1-2 ; f, 4)$

Großvater:

Ns-Jmn-nst- $t 3 w j$

--- $(a, 4 ; f, 4)$

Urgroßvater:

[Wsr-h3t]-msjw

Ururgroßvater:

[ ] $-y$

$$
\begin{aligned}
& w^{e} b(\mathrm{f}, 4) \\
& h r j \text { sšt3 }(\mathrm{f}, 4) \\
& h r j \text { zš qdwt(?) n pr Jmn (f,5) } \\
& \text { p3 k3nw(?) p3 wb3 } 3 \text { [ ] (f,5) }
\end{aligned}
$$

\footnotetext{
17 Ähnliche Dekorationsmuster gibt es auf zahlreichen Würfelhockern der Dritten Zwischenzeit, vgl. W. Wreszinski, OLZ 18 (1915), p. 355-6.

18 Der Grund dafür ist nicht ersichtlich. Man hat fast den Eindruck, als sei die Figur, die ja sehr klein ist, als Reibstein benutzt worden, um irgendetwas zu polieren.

${ }_{19}$ B.V. Bothmer, MDAIK 37 (1981), p. 80, n.25 (als eine vom Enkel gestiftete Statue).
} 
Sohn:

Wsr-h3t-msjw

dd.tw n.f Jpw-wr

Enkel (und Stifter der Statue):

Dd-B3stt-jw.f- $n h$

$[h m(?)]-n \underline{t r}[\ldots m] J p t-s w t(f, 1)$

$z \check{s} q d w t m m n w n b n[\operatorname{pr}(?)] \operatorname{Jmn}[\ldots](\mathrm{f}, 2)$

Diese auf der Statue erwähnten Familienmitglieder (s.u.) ergeben folgenden Stammbaum:

a) Oberseite (Fig.7)

1 Der Wab-Priester mit Zutrittsrecht in Karnak, 2 der Aktenschreiber(?)(1) Dd-Hnzw, gerechtfertigt, 3 der Sohn des Jpw-wr(2), gerechtfertigt, 4 der Sohn des Ns-Jmn-nst-t3wj(3), gerechtfertigt.

b) Vorderseite (Fig.8)

- Über dem Heck der Barke: Dd-Hnzw-jw.f- $n h$, der Sohn des Wsr-ḩ3t-msjw.

- Über dem Bug: (Sokar) folgen(4).

c) Rechte Seite (Fig.8)

1 Der Osiris, der Wab-Priester mit Zutrittsrecht in Karnak, der Schreiber [der Akten $\underline{D} d$ $H n z w\left(j w . f-{ }^{e} h h\right.$ ? )], gerechtfertigt, der Sohn des Jpw-wr, 2 indem er sagt: O [alle] L[eute von Karnak, die komm]en werden danach(5), (meine) Kollegen darunter gleichfalls(6): 3 Gedenkt meines Ka, seid nicht taub gegen mich, damit eure Namen (ihrerseits dauernd) auf 


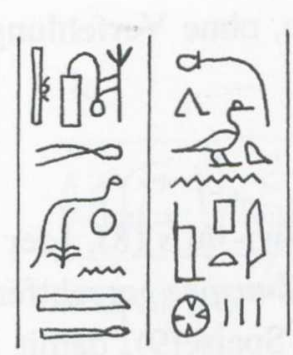

a) Oberseite

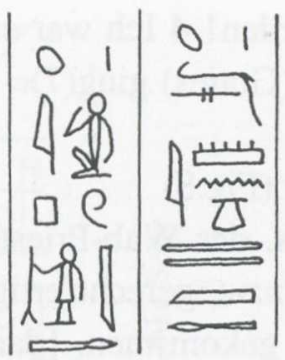

e) Um die Arme

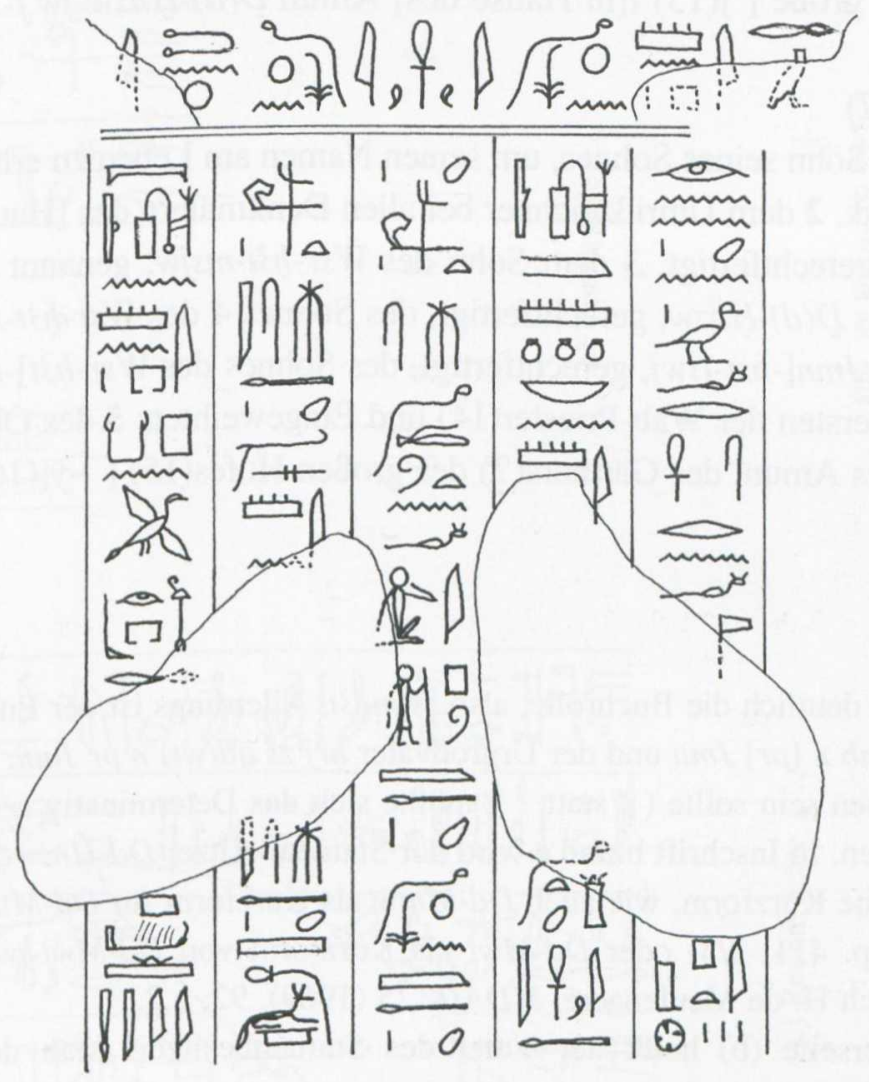

f) Rückseite

Fig. 7: Kairo JE 37365 
Erden sein werden! 4 Ich war einer mit gerechtem Herzen, ohne Verfehlung, der aufrecht auf dem Weg (Gottes) ging(7).

\section{d) Linke Seite (Fig.8)}

1 Der Osiris, der Wab-Priester im Monatsdienst von $J w j-3 h[. s](8)$, [der Schreiber der Akten?] Dd-Hnzw, gerechtfertigt, der Sohn des Wsr-h3t-2-msjw, gerechtfertigt, indem er sagt: [Ich bin gekom]men, [damit] (ich) [ess]e(?) deine Speise(9), damit ich in deinem Tempel bin, 3 Amun, Urzeitlicher der Beiden Länder! (Denn) Du bist der Herr der Tempel, deine Macht hat sie gegründet. Mögest du mir die Opferbrote gewähren, 4 die dargebracht werden auf deinem Opfertisch in Karnak, indem (mein) Sohn (dann seinerseits) tut, was (ich) getan habe(10), von Sohn zu Sohn(11) in Ewigkeit.

e) Um die Arme (Fig.7)

rechts: $[\ldots]$ der Sonne(12) $\underline{D}(d)-H n z w-j w . f-n h$.

links: [... ] der große [ ](13) i[m Hause des] Amun $\underline{D}(d)-H n z w-j w . f-{ }^{e} n h$.

\section{f) Rückseite (Fig.7)}

1 Gemacht vom Sohn seines Sohnes, um seinen Namen am Leben zu erhalten, dem Pro[pheten(?) ... in] Karnak, 2 dem Umrißzeichner bei allen Denkmälern des [Hauses] des Amun [...] Dd-B3stt-jw.f- $n h$, gerechtfertigt, 3 dem Sohn des Wsr-ḩ3t-msjw, genannt Jpw-wr, gerechtfertigt, des Sohnes des $\underline{D}(d)$ - $H n z w$, gerechtfertigt, des Sohnes 4 des Wsr-ḩ3t-msjw, gerechtfertigt, des Sohnes des Ns-Jmn[-nst-t3wj, gerechtfertigt, des Sohnes des Wsr-h3t]-msjw, gerechtfertigt, des Sohnes des Obersten der Wab-Priester(14) und Eingeweihten, 5 des Obersten Umrißzeichners des Hauses des Amun, des Gärtners(?) des großen Hofes(15) [ ]-y[(16), gerechtfertigt.

\section{ANMERKUNGEN}

1) Geschrieben ist deutlich die Buchrolle, also $z \check{s} m \underline{m} 33 t$. Allerdings ist der Enkel dieses Mannes $z \check{S}$ $q d(w t) m m n w n b n[p r] J m n$ und der Urgroßvater hrj žs qd(wt) $n$ pr Jmn. Falls daher auch hier $z \check{s} q d(w t)$ zu lesen sein sollte ( $\}$ statt $\}$ ), müßte sich das Determinativ $\nsim$ auf die Gesamtverbindung beziehen. In Inschrift b und e wird der Statuenbesitzer $\underline{D} d-H n z w$-jw.f- $n h$ genannt, $\underline{D} d$ $H n z w$ ist nur eine Kurzform, wie auch $\underline{D} d-M n t w$ als Kurzform für $\underline{D} d-M n t w$-jw $f$ - $n h$ belegt ist (Ranke, $P N$ I, p. 411, 2/3) oder $\underline{D} d-M w t$ als Kurzform von $\underline{D} d-M w t$-jw.s- $n h$ (ibid., p. 410, 14/16). Vgl. auch H. de Meulenaere, MDAIK 25 (1969), 92, n.2.

2) Auf der Vorderseite (b) heißt der Vater des Statuenbesitzers statt dessen Wsr-h3t-msjw, ebenso in d,1-2 und f,4, dagegen in c,1 wiederum Jpw-wr. Der Sohn des Statuenbesitzers führt ebenfalls diese beiden Namen; er wird in f,3 explizit als Wsr-h3t-msjw mit dem Beinamen (dd.tw $n . f$ ) $J p w$-wr eingeführt. Dabei wird man auch beim Vater annehmen dürfen, daß 


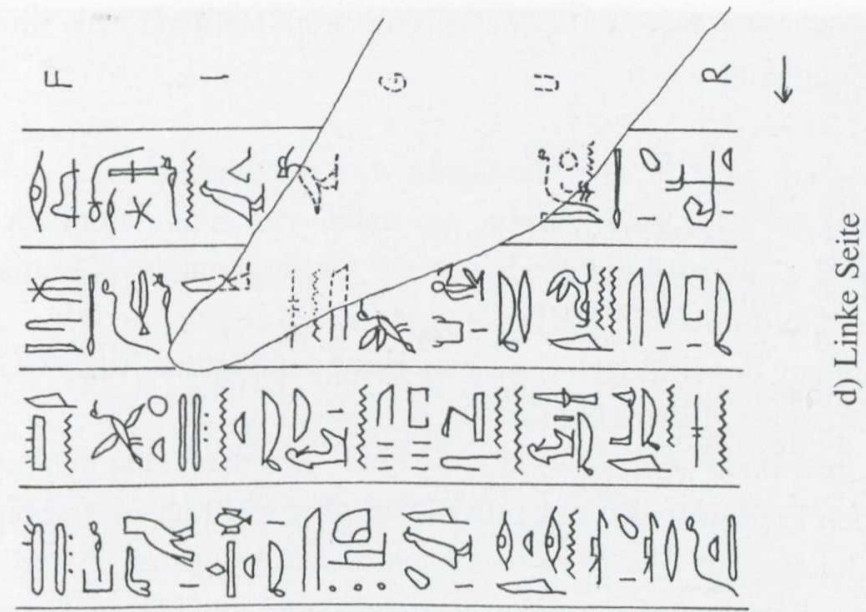

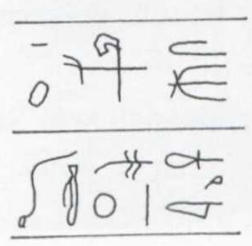

एा

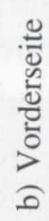

$\infty$

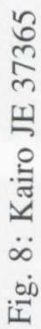

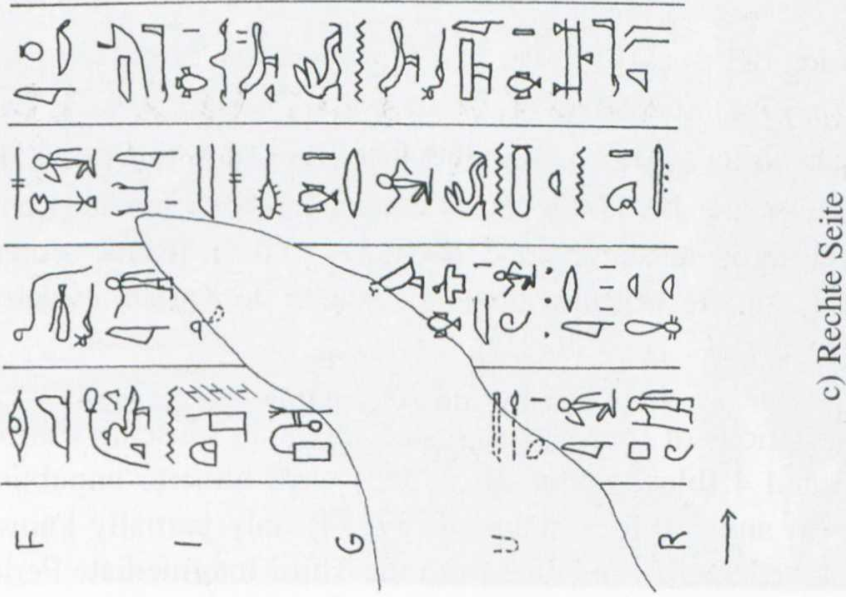


$J p w$-wr sein Beiname war (obwohl die Verteilung von 'Hauptnamen' und 'Beinamen' oft recht willkürlich gehandhabt wird).

3) S. Ranke, $P N$, I, p. 174,1 .

4) In Hieroglyphen steht nur šmsj da, aber die Barke wird mitzulesen sein.

5) Vielleicht zu 参 $[$ 过。 Anruf an die rmtw nt Jpt-swt auch auf der Statue der Gottesgemahlin Karomama im Louvre (N 500), vgl. H. Jacquet-Gordon, ZÄS 94 (1967), p. 90 (Z.2).

6) Eine ähnliche Erwähnung der Kollegen ( $g$ sjw) auch auf den Statuen Kairo CG 42207, f,2-3 ( $\ddot{A} g$. Biogr., p. 450) und 42208, c,14 (ibid., p. 455).

7) So verstanden muß man šm $h r w 3 t^{\circ} q 3(w)$ lesen, wobei $₫$ dann eine Schreibung des Partizips von $\breve{m}$ wäre (vgl. den kopt. Infinitiv $\mathbf{c y \epsilon}$ ). Denkbar, aber wohl unwahrscheinlicher, wäre auch šm.j hrr w3t ${ }^{c} q 3(. j)$, ich bin auf dem Weg gegangen, indem ich aufrecht war".

8) Zu ähnlich gebildeten Titeln auf den Statuen Kairo JE 36971 und JE 36998 und zu dieser Ortsbezeichnung vgl. K. Jansen-Winkeln, Biographische und religiöse Inschriften der Spätzeit, p. $32(10)$.

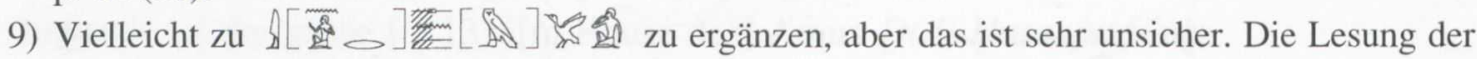
Reste über dem $\mathfrak{H}_{\text {S }}$ als $\underset{\cdots}{\rightleftharpoons}$ ist nur eine vage Möglichkeit.

10) Wohl jw z3(.j) <hr $>$ jrt jrj.n(.j) zu lesen. Gemeint ist zweifellos, daß sein Sohn den Totenkult für ihn aufrechterhalten soll, so wie er es für seine Väter getan hat.

11) Zum Ausdruck $w^{e}-z 3-w^{e}$ s. Jansen-Winkeln, GM 123 (1991), 53-6.

12) Unmittelbar vor dem Namen sollte man an sich das letzte Element eines Titels erwarten, aber ein Titel, der auf $j$ tn endet, erscheint doch zweifelhaft. Es dürfte sich eher um ein Beiwort handeln, vgl. etwa Äg. Biogr., p. 482 (f): $m 33$ jtn (jmj W3st).

13) Vielleicht $\left[w^{c} b\right]{ }^{c} 3 \mathrm{~m}[\mathrm{pr}] \mathrm{Jmn}$ o.ä.?

14) $\square$ sicher ein Fehler für $\square$.

15) Ein Titel $p 3 k 3 n w p 3 w b 3^{\circ} 3$ (falls so zu lesen ist) scheint nicht bekannt zu sein.

16) Das Doppelschilfblatt kann hinter dem Determinativ nur die „Koseendung“ sein, vgl. Ranke, $P N$, II, p. 143ff.

\section{Résumé / Abstract}

Publication des inscriptions de quatre statues du Musée du Caire. Les textes de la statue-cube JE 36926 (no.1) et de la statue-cube JE 37365 (no.4) étaient jusqu'ici inédits, ceux de la statue agenouillée JE 36943 (no.2) et de la statue-cube JE 37374 (no.3) étaient seulement partiellement connus. Ces quatre statues ont été trouvées dans la cachette de Karnak et datent de la Troisième période intermédiaire.

Publication of the inscriptions of four statues in the Egyptian Museum Cairo. The texts of no. 1 (block statue JE 36926) and 4 (block statue JE 37365) were hitherto unpublished, those of no. 2 (kneeling statue JE 36943) and 3 (block statue JE 37374) only partially known. All four statues were found in the Karnak "cachette" and date from the Third Intermediate Period. 


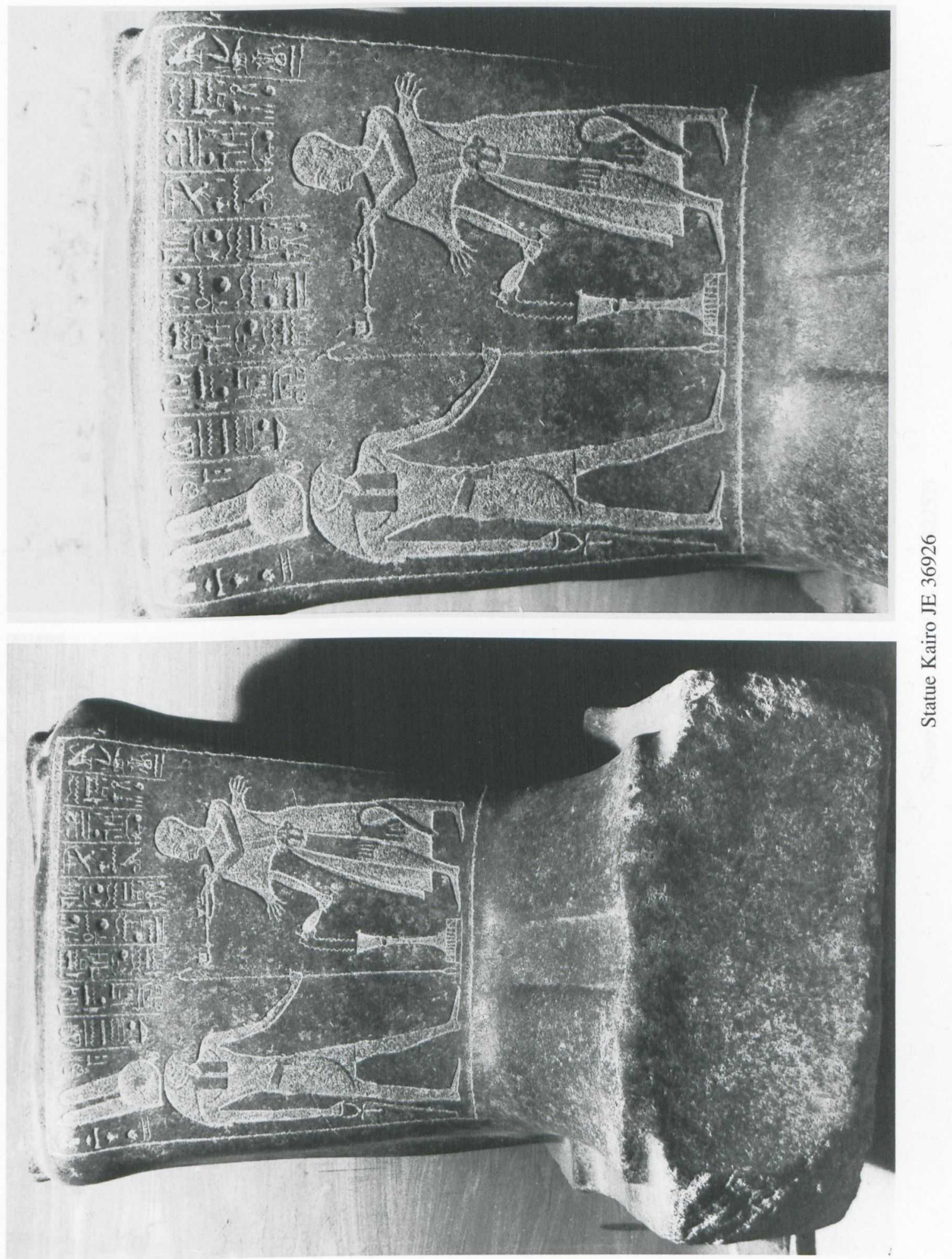

$\mathrm{K}$. Jansen-Winkeln, Zu einigen Inschriften der Dritten Zwischenzeit. 


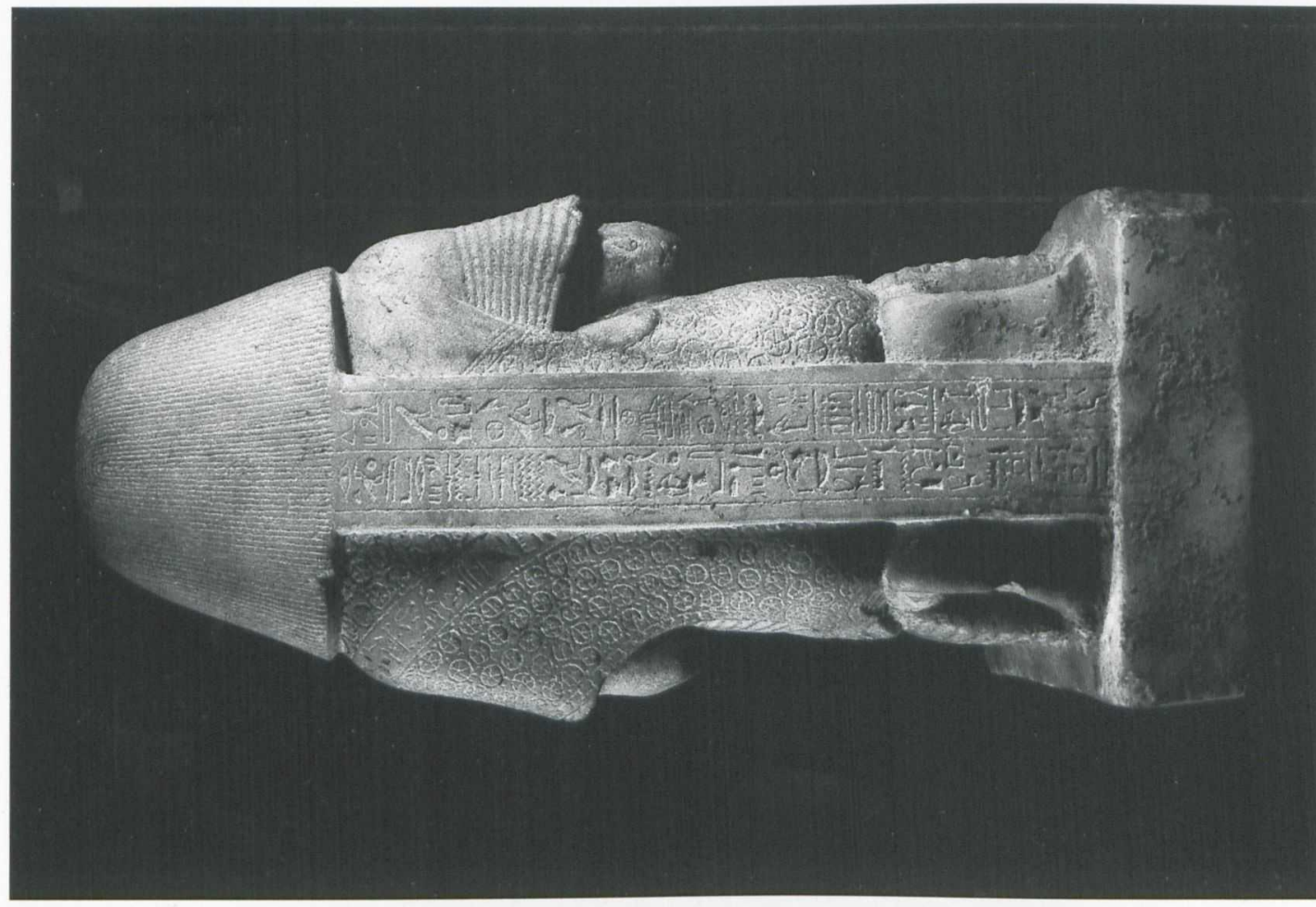

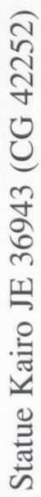

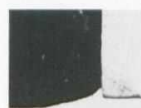

.)

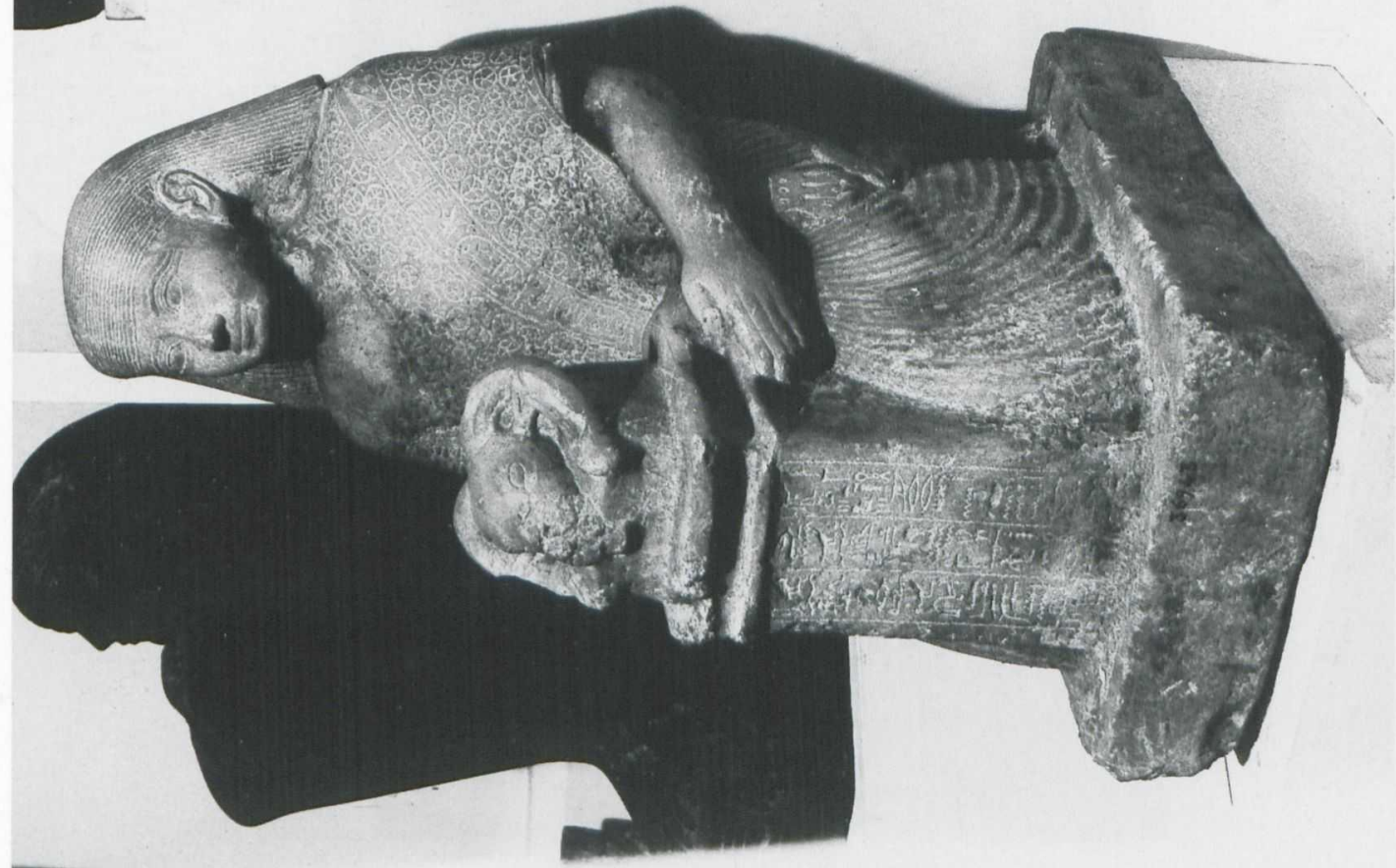

$\mathrm{K}$. Jansen-Winkeln, Zu einigen Inschriften der Dritten Zwischenzeit. 


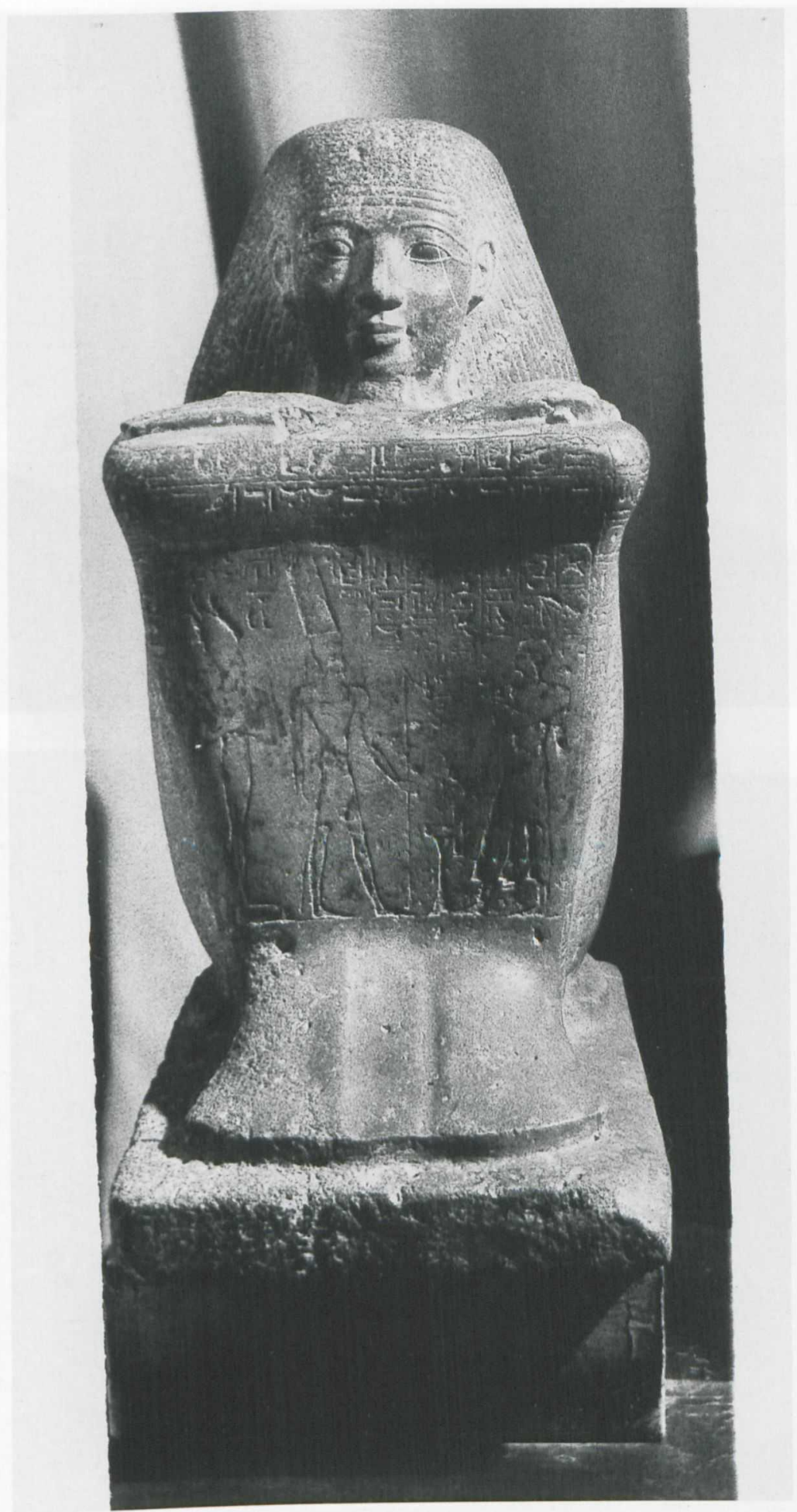

Statue Kairo JE 37374 (CG 42254)

K. Jansen-Winkeln, Zu einigen Inschriften der Dritten Zwischenzeit. 


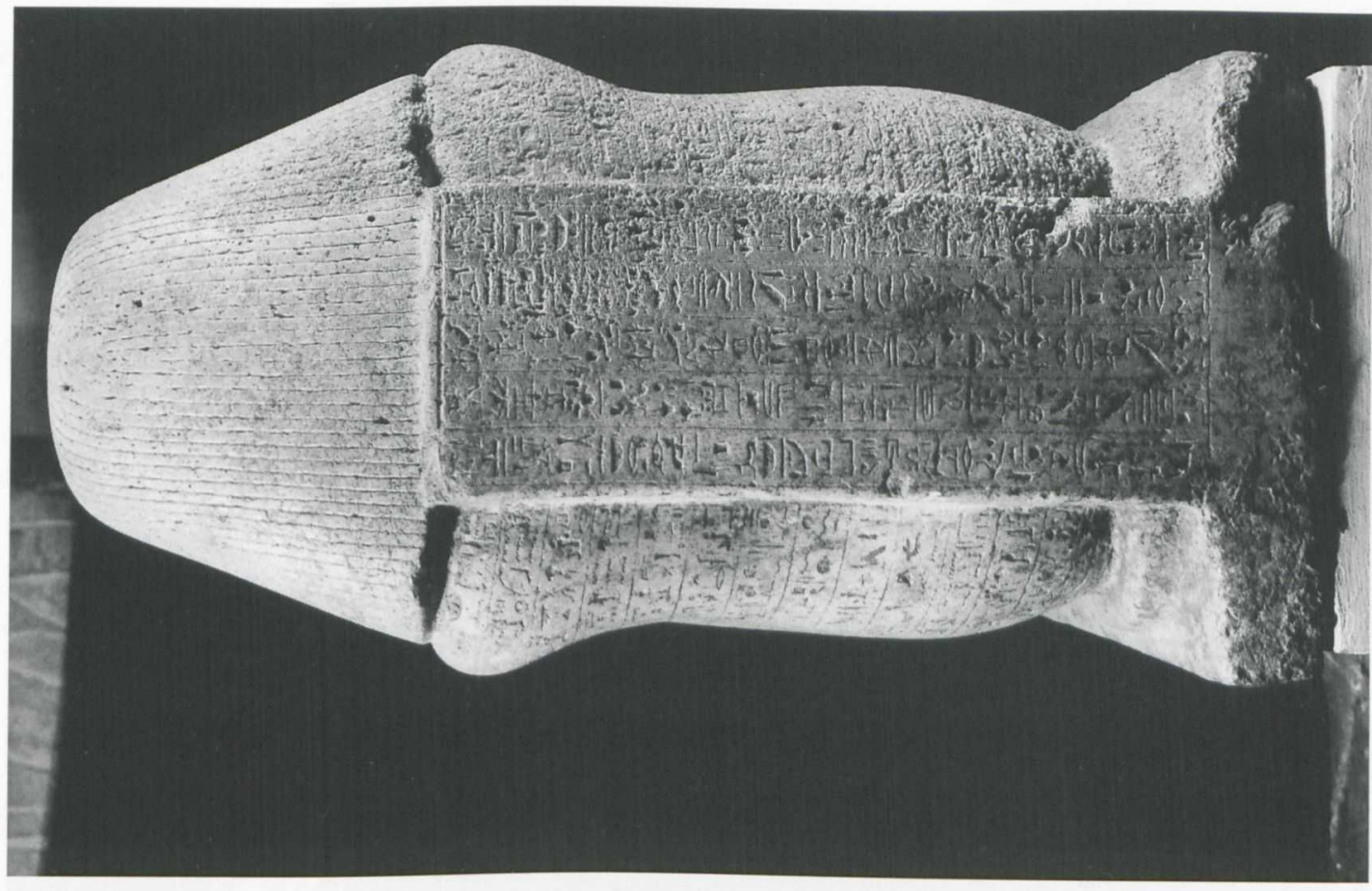

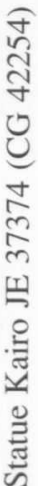

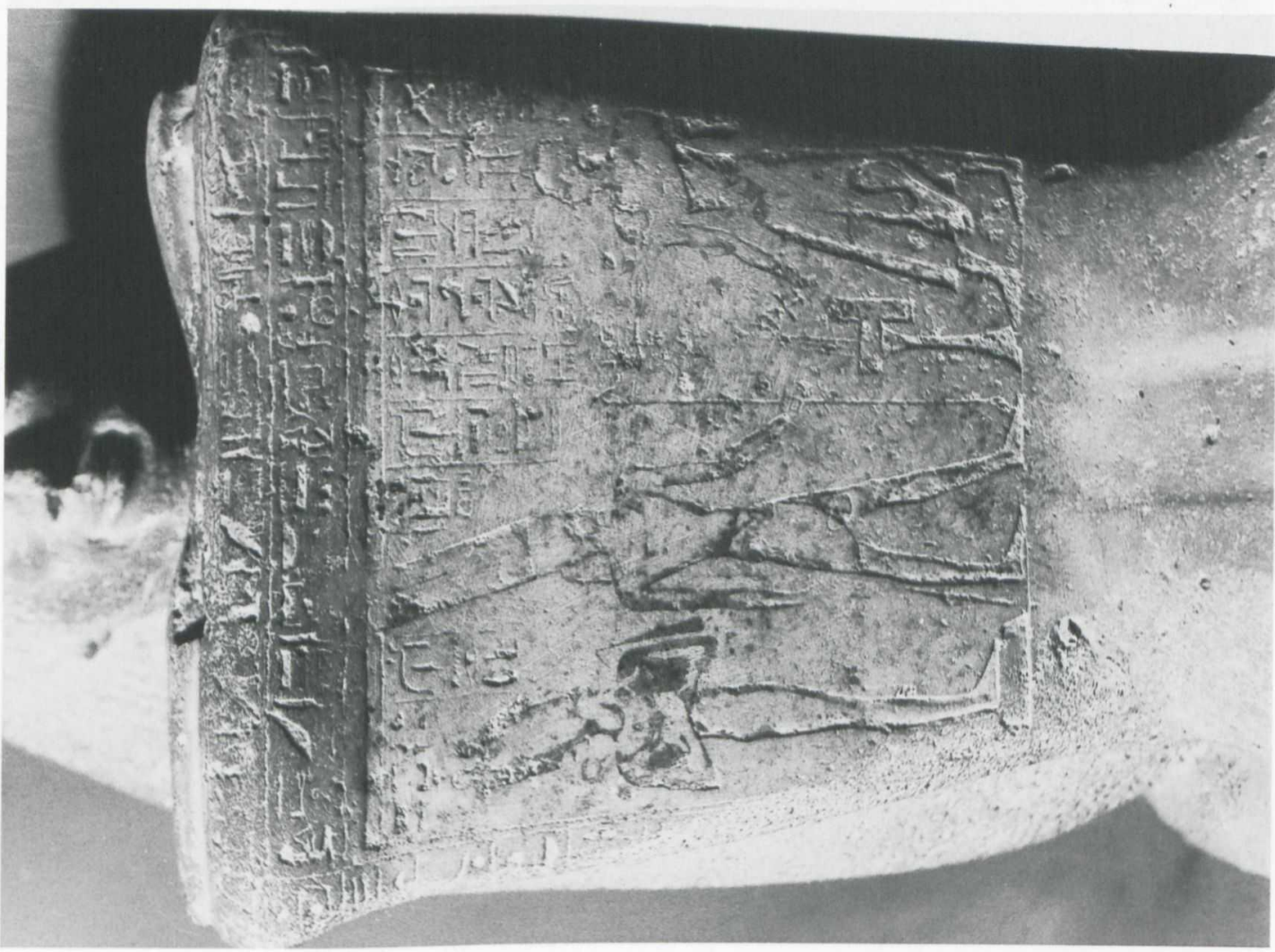

K. Jansen-Winkeln, Zu einigen Inschriften der Dritten Zwischenzeit. 


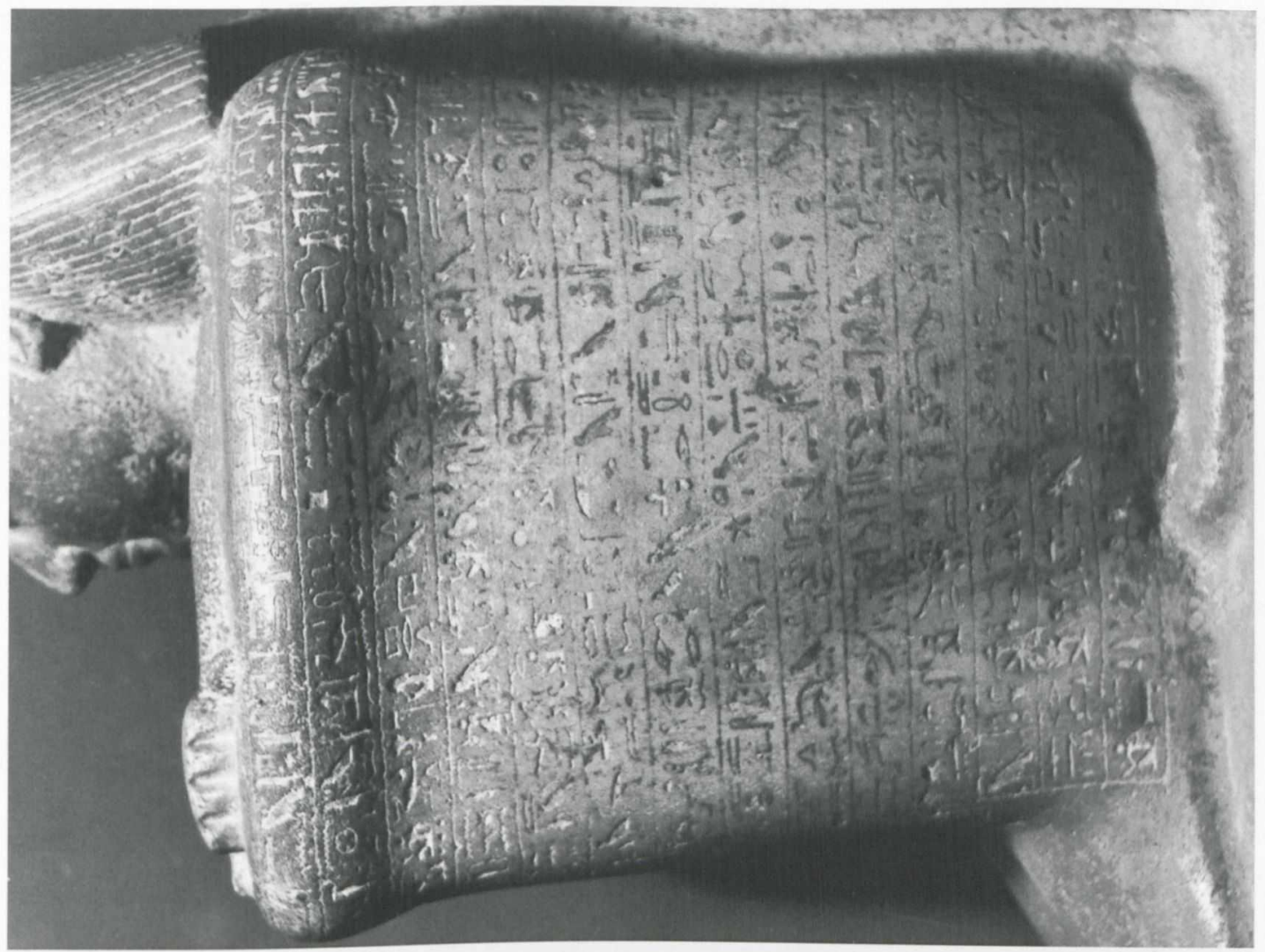

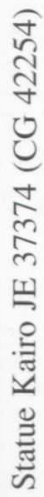

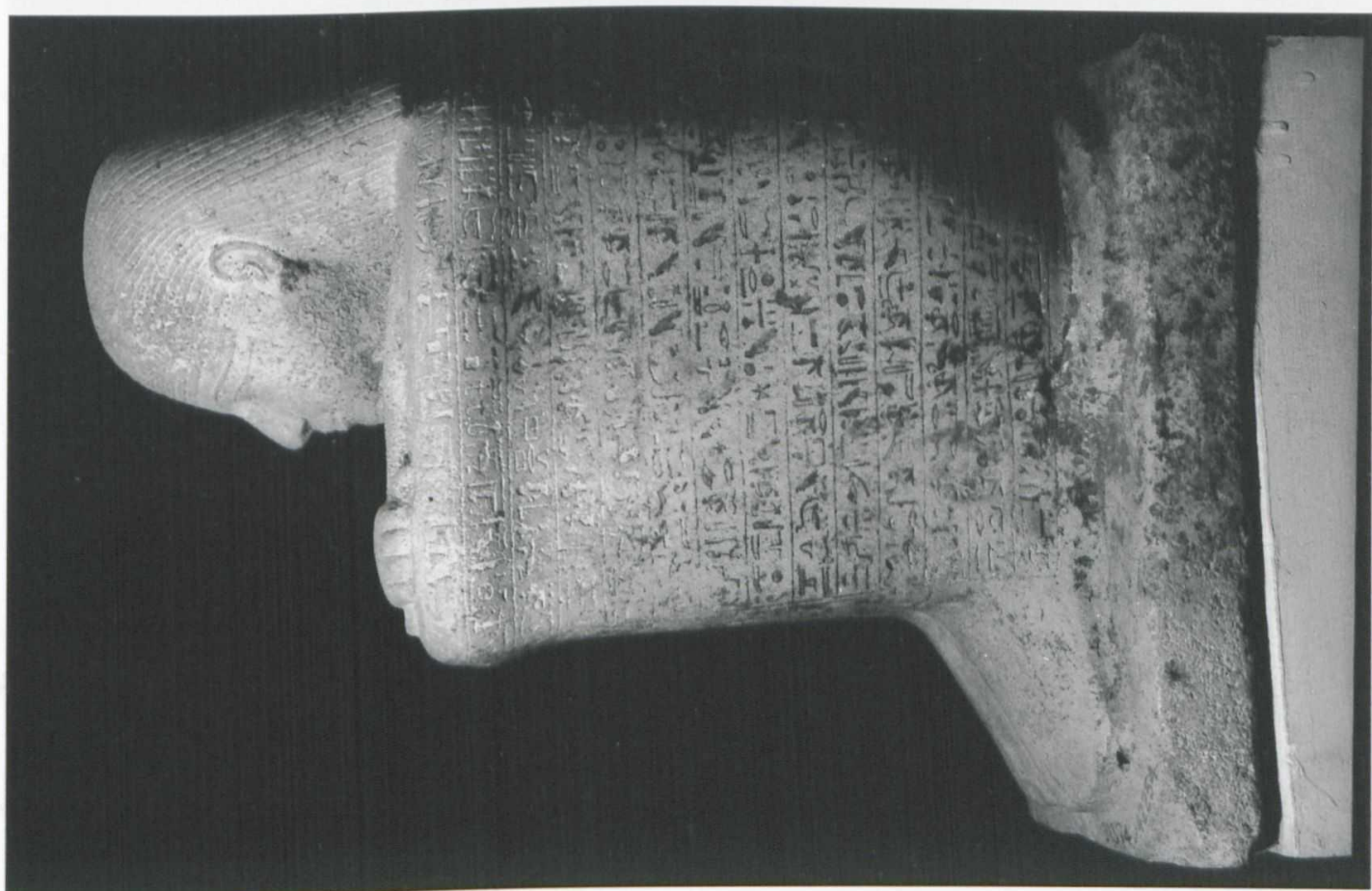




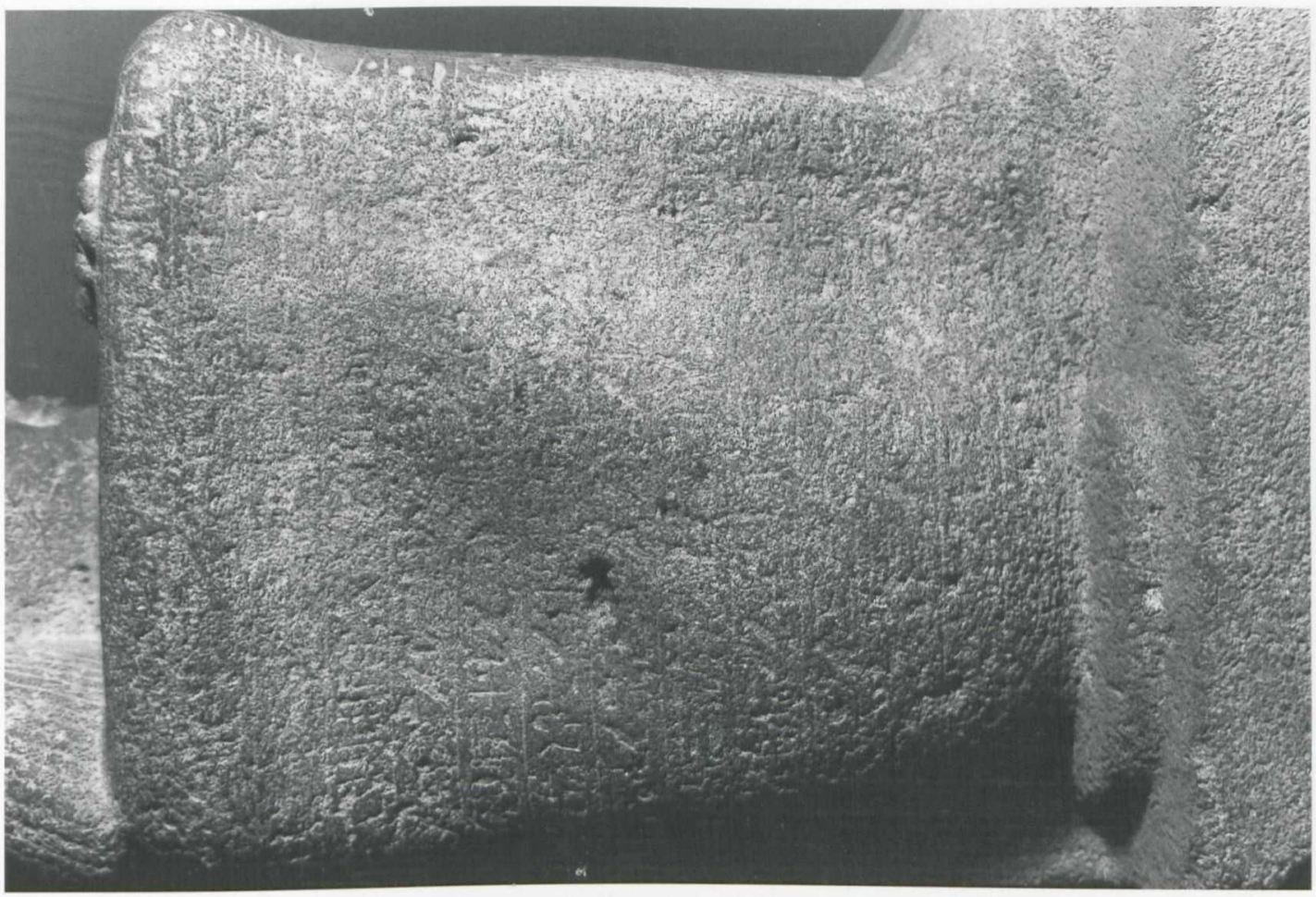

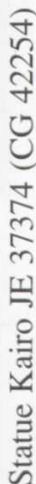

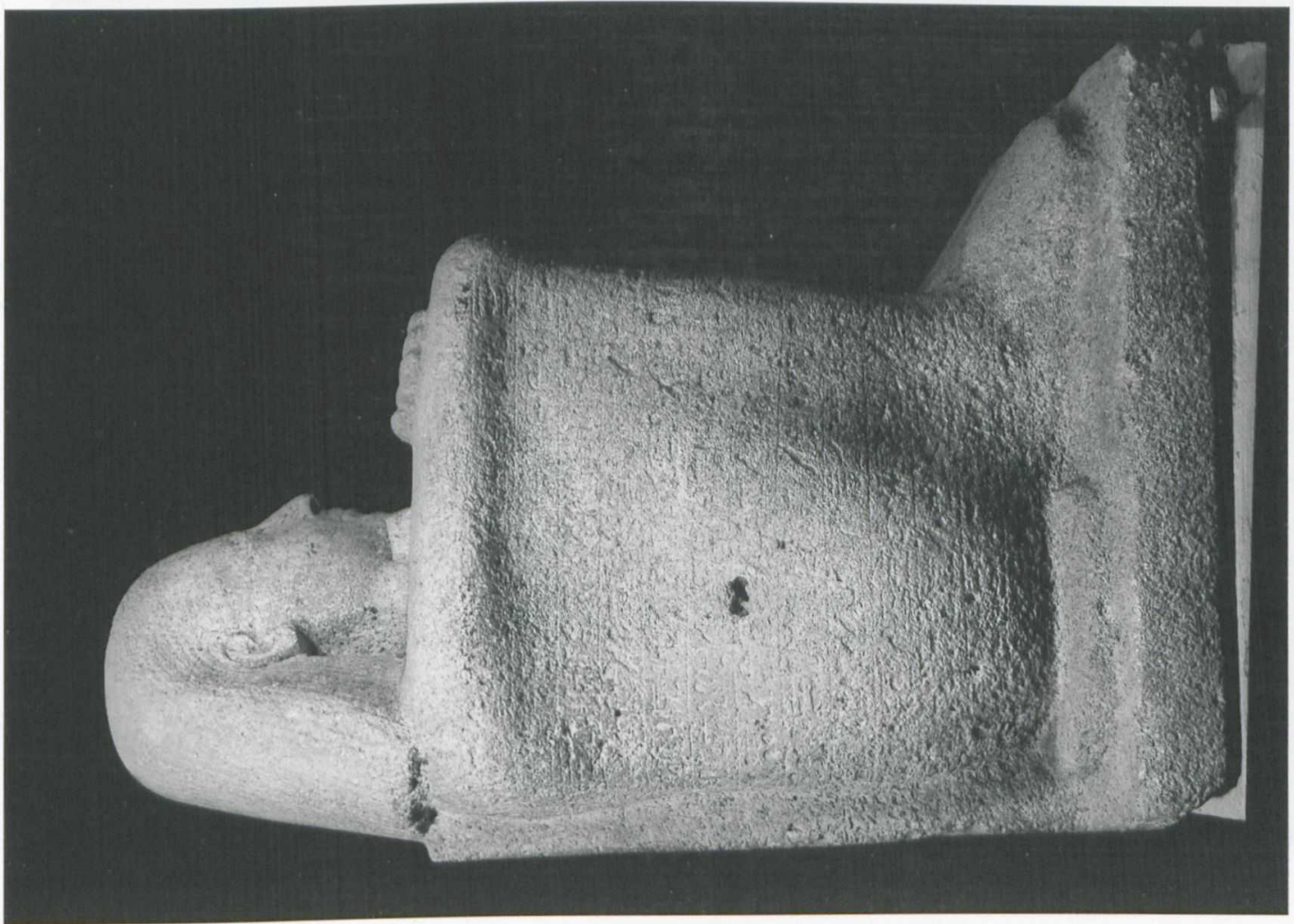

K. Jansen-Winkeln, Zu einigen Inschriften der Dritten Zwischenzeit. 

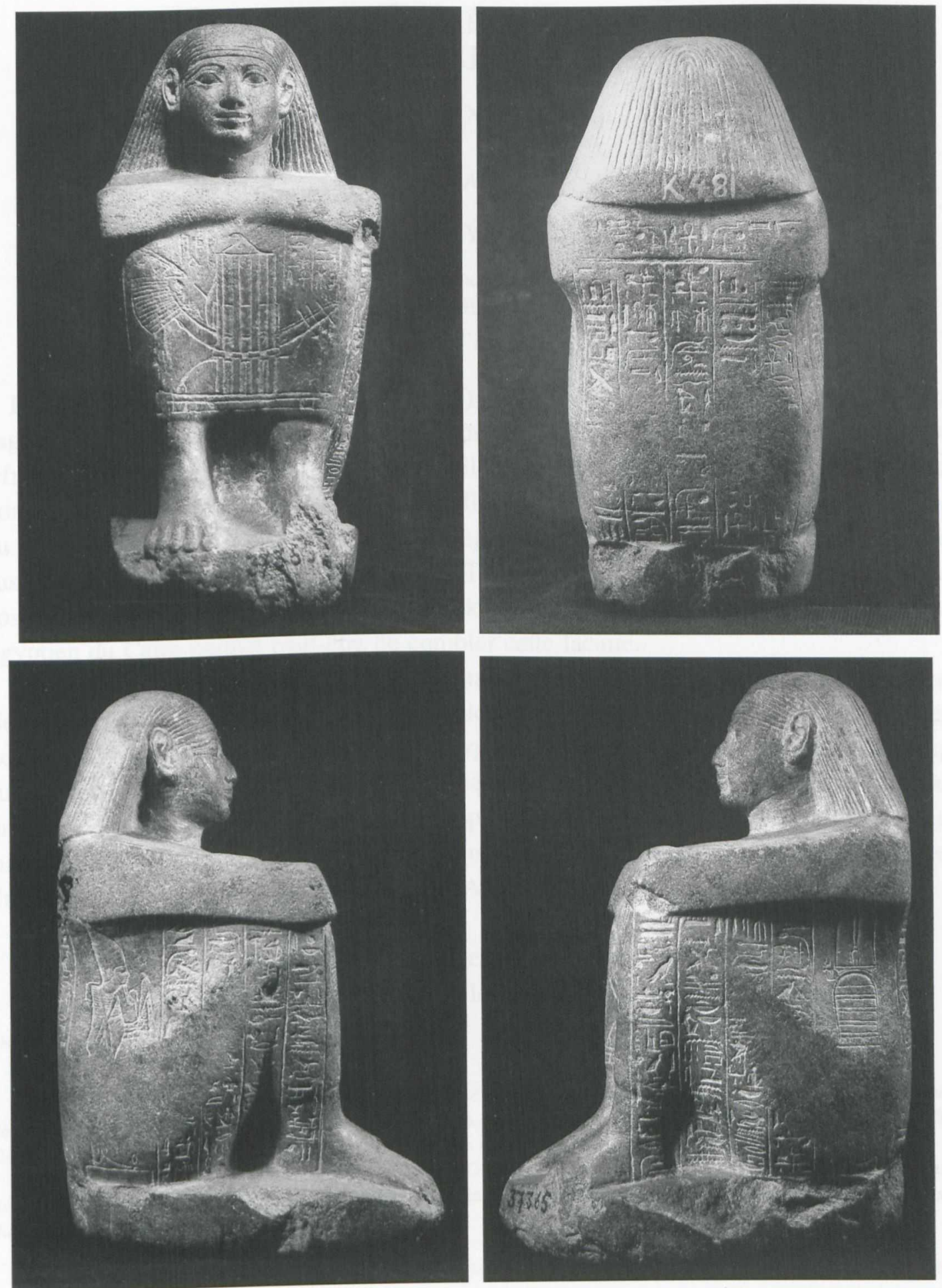

Statue Kairo JE 37365

K. Jansen-Winkeln, Zu einigen Inschriften der Dritten Zwischenzeit. 